\title{
VARIABILITY OF PRACTICE AND STRENGTH TRAINING PERIODIZATION: WHEN THEORIES COLLIDE
}

\author{
A Thesis \\ presented to \\ the Faculty of California Polytechnic State University, \\ San Luis Obispo
}

\begin{abstract}
In Partial Fulfillment
of the Requirements for the Degree

Master of Science in Kinesiology
\end{abstract}

by

Katherine Lauren Streder

December 2013 
(C) 2013

Katherine Lauren Streder

ALL RIGHTS RESERVED 
TITLE:

Variability of Practice and Strength Training Periodization: When Theories Collide

AUTHOR:

Katherine Lauren Streder

DATE SUBMITTED:

December 2013

COMMITTEE CHAIR:

Kellie G. Hall, Ph.D.

Professor of Kinesiology

COMMITTEE MEMBER: Todd Hagobian, Ph.D.

Professor of Kinesiology

COMMITTEE MEMBER: Jennifer Davis Olmstead, MS, CSCS

Full Time Lecturer of Kinesiology 


\begin{abstract}
Variability of Practice and Strength Training Periodization: When Theories Collide

Katherine Lauren Streder
\end{abstract}

The primary purpose of this study was to examine whether variability of practice enhances neuromuscular adaptations when compared to traditional strength training. The secondary purpose was to investigate whether there was a difference in perception of exertion between the two strength programs. Forty one subjects ( 23 women and 18 men) were assigned to either the control group or one of two treatment groups by a blockedrandom method. Subject's one repetition maximum (1RM) for the kettlebell press and leg press were measured at baseline, after 4 weeks of training, and after 8 weeks of training. The treatment groups completed 8 weeks of training consisting of 3 days a week with at least one recovery day in between each session. In weeks 1 through 3 , subjects undergoing the traditional treatment completed 3 sets of 8 repetitions at $70 \%$ of their $1 \mathrm{RM}$ for each exercise. Weeks 4 through 6 were composed of 3 sets of 6 repetitions at $77 \% 1 \mathrm{RM}$. In weeks 7 and 8, 3 sets of 4 repetitions at $85 \% 1 \mathrm{RM}$ were performed. Over the course of the eight weeks, the variable treatment was comprised of 5 sets of the kettlebell and leg press. A set of 4 repetitions at $77 \%$ of the subject's 1 RM, one of 5 repetitions at $70 \% 1 \mathrm{RM}$, another of 3 repetitions at $85 \% 1 \mathrm{RM}$, one of 6 repetitions at $65 \% 1 \mathrm{RM}$, and finally a set of 2 repetitions at 93\% 1RM were performed every session. The order of these sets were changed every 3 weeks, altering where in the session the highest intensity set was in relation to the lower intensity sets. Ratings of perceived exertion (RPE) were recorded following treatment to determine subject's perception of intensity during each set. Analysis of variance with repeated measures did not reveal significantly different strength gains between treatment groups in either lift, although variable treatment resulted in greater mean strength gains over almost every time interval. Analyses of RPE data revealed significantly lower reported RPE values for the variable treatment compared to the traditional treatment in both lifts. The greater mean strength gains and significantly lower RPEs of the variable treatment program compel us to conclude it is a superior training method for increasing strength compared to the traditional program. 


\section{ACKNOWLEDGMENTS}

I would like to thank my entire committee for all the support and constant guidance over the past two years in developing and implementing my thesis. Without any of them, I would not be where I am today. Dr. Todd Hagobian is the professor that sparked my love of exercise physiology and inspired me to complete my own Master's thesis. His knowledge and expertise in exercise physiology is a major reason I strive to continue my professional development in the field. Jennifer Olmstead is the only instructor in the Kinesiology department with substantial knowledge in the area of strength training and the experience to help me design a strength training study. Her encouragement and continuous interest in my thesis kept me on course and able to overcome the many encountered obstacles. As a Kinesiology student the statistical analyses required to make valid interpretations of my data proved an area of weakness for me. Dr. Steve Rein consulted me and helped interpret results on top of all his other university responsibilities. I am forever grateful for his expertise and patience. Dr. Kellie Hall was my pillar of strength over the past two years. Thesis related or not, Dr. Hall's investment in my success motivated me throughout my graduate education. Her genuine concern and desire for students to get the most out of their education is rare and should be honored much more than it is presently in higher education. Dr. Hall is a gem among her fellow Ph.D.s and should be recognized as such.

A special thanks to Sleeping Tiger Fitness and Nathan Zimmerman is warranted as I was graciously allowed to use his facility for data collection. Additionally, the underlying idea that spawned the present study was originally proposed by Nathan to Dr. Hall. His help with the study design and data collection was essential and deserves more than just a thank you. Finally, I would like to make sure my partner in the hell that is doing a thesis, Amanda Morris, gets the credit she deserves for her part in the completion of my thesis. The endless hours of coffee shop hopping and working on our theses together was one of very few happy memories of the experience. Without her, I fear I would not have made it out of graduate school alive.

Finally, without the emotional support and financial investment from my parents I wouldn't have gone to graduate school and been able to complete a Master's thesis. I can now officially say I am their most expensive child and will now make it my goal to be worth every penny. Thank you mom and dad, I love you. 


\section{TABLE OF CONTENTS}

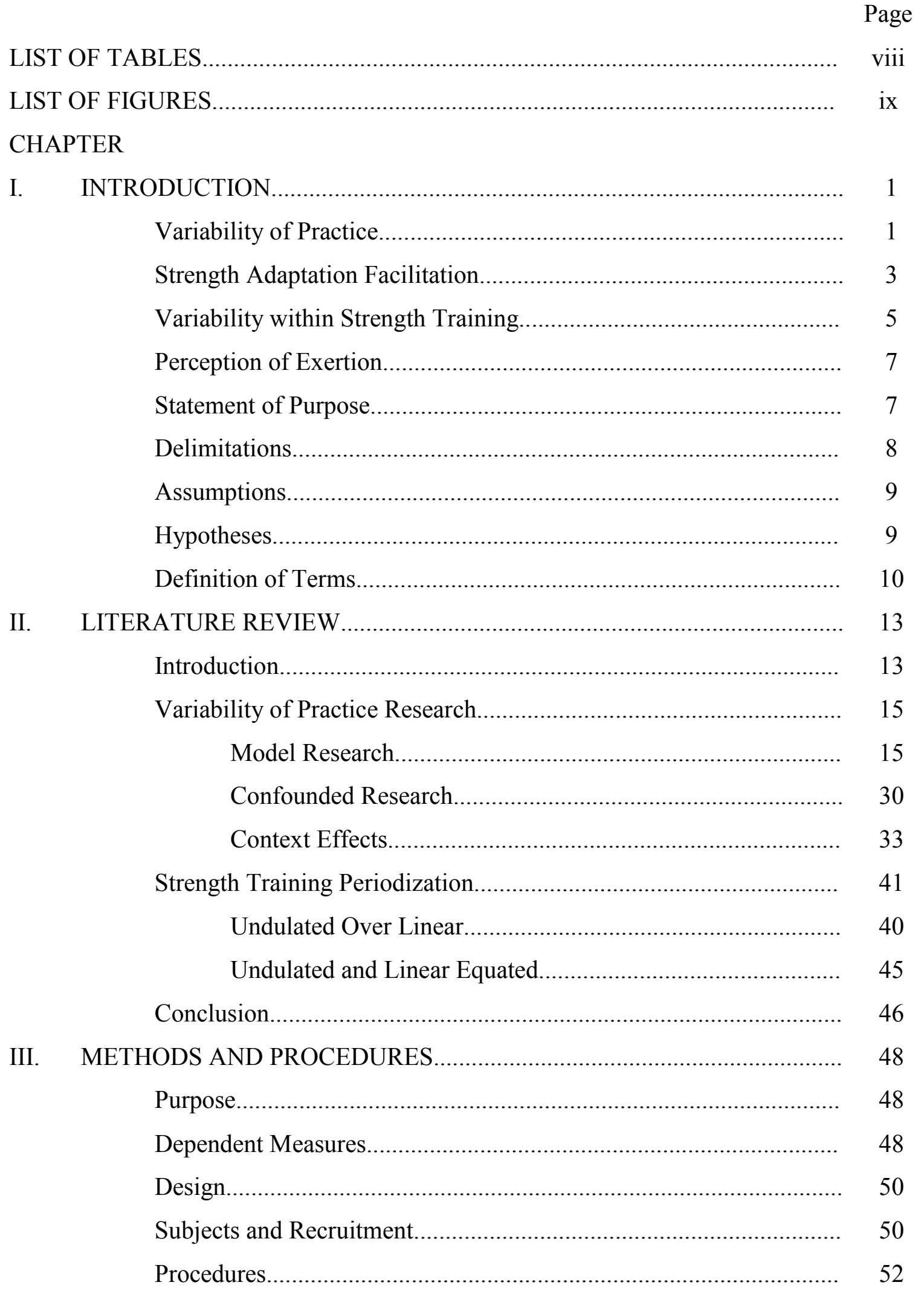


Statistical Analyses................................................................. 54

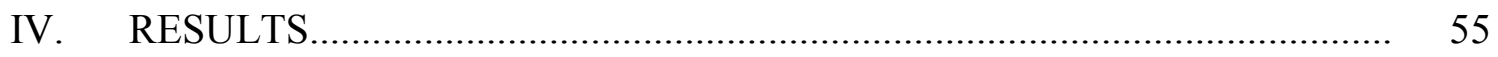

Restatement of Purpose and Hypotheses........................................... 55

Dependent Measures..................................................................... 55

Subject Characteristics.................................................................. 56

Time Effects on Strength.............................................................. 57

Treatment Effects on Strength.......................................................... 59

Ratings of Perceived Exertion Effects and Responses........................ 63

Sex Differences.......................................................................... 66

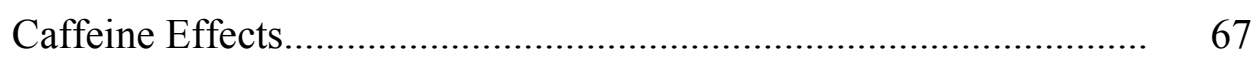

V. DISCUSSION AND CONCLUSIONS.................................................... 69

Strength Gains.......................................................................... 71

Ratings of Perceived Exertion........................................................ 72

Sex Differences....................................................................... 75

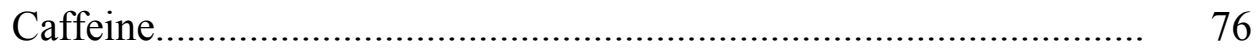

Related Literature...................................................................... 77

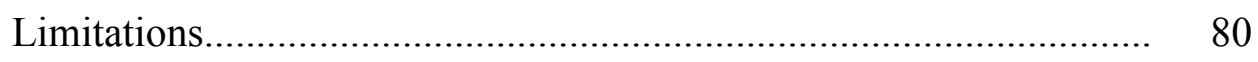

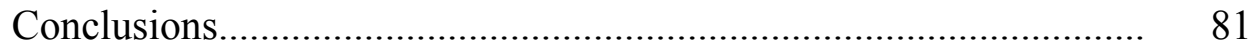

Recommendations....................................................................... 82

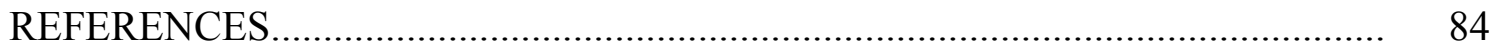




\section{LIST OF TABLES}

Table Page

3.1 Training programs defined by specific set and repetition schematics........ 53

4.1 Subject characteristics................................................................. 57

4.2 Mean 1RM (kg) measures and relative strength (1RM/BW) over time..... 59

4.3 Mean absolute and percent strength gains (change in 1RM) across time... 61

$4.4 \quad$ Repeated Measure ANOVA Alpha Levels............................................. 63

4.5 Alpha levels of RPE effects on strength gains for treatment groups.......... 64

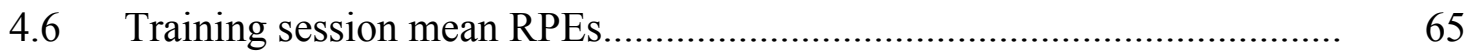

4.7 Strength gains and relative strength increases by sex................................ 67

4.8 Alpha levels of caffeine use within 4 hours of training sessions................. 68 


\section{LIST OF FIGURES}

Figure $\quad$ Page

4.1 Absolute strength gains made in the kettlebell press by all groups.

Values are changes in 1RMs $(\mathrm{kg})$ over the first 4 weeks of training (I1), the second 4 weeks of training (I2) and the entire 8 weeks (I3)..............

4.2 Absolute strength gains made in the leg press by all groups. Values are changes in 1RMs (kg) over the first 4 weeks of training (I1), the second 4 weeks of training (I2) and the entire 8 weeks (I3). *Significantly (p $<.05)$ greater gains compared to the control group................................

4.3 Percent strength gains made in the kettlebell press by all groups. Values are changes in 1RMs (kg) divided by baseline 1RM values and multiplied by 100, over the first 4 weeks of training (I1), the second 4 weeks of training (I2) and the entire 8 weeks (I3). * Significantly (p $<.05)$ greater gains compared to the control group.

4.4 Percent strength gains made in the leg press by all groups. Values are changes in 1RMs (kg) divided by baseline 1RM values and multiplied by 100 , over the first 4 weeks of training (I1), the second 4 weeks of training (I2) and the entire 8 weeks (I3). * Significantly $(\mathrm{p}<.05)$ greater gains compared to the control group.

4.5 Mean reported ratings of perceived exertion (RPE) for the kettlebell press over both 8 week treatments. Values are based on a scale of 1 to 10,10 being maximum exertion. *Significantly $(\mathrm{p}<.05)$ lower reported RPEs.

4.6 Mean reported ratings of perceived exertion (RPE) for the leg press over both 8 week treatments. Values are based on a scale of 1 to 10,10 being maximum exertion. ${ }^{*}$ Significantly $(\mathrm{p}<.05)$ lower reported RPEs 


\section{CHAPTER I}

\section{Introduction}

\section{Variability of Practice}

After 30 years of empirical examination since its conceptual manifestation, the Variability of Practice Hypothesis is a well established premise in the motor learning and control literature. Derived from Schmidt's Schema Theory, the Variability of Practice Hypothesis suggests greater learning of new motor tasks occurs when multiple versions of a task are practiced concurrently as opposed to one at a time (Hall, 1990; Schmidt, 1975; Schmidt, 1988; Schmidt and Lee, 2005). Schmidt's Schema Theory (1975; 1988) hypothesized that habitual practice of a skill results in the formation of a cognitive rule, or schema. This abstract rule is stored in memory and acts as a control center for information processing related to initiation and modification of motor skills. One such rule, coined the motor response schema, is especially important for understanding the concept of Variability of Practice. Motor skills with similar form and function are governed by what Schmidt (1975) referred to as a generalized motor program or GMP. Execution of a motor skill requires contextual parameters to be cognitively "programmed" in to the GMP of a motor skill depending on the desired outcome. Parameters can be set for limb choice, absolute force and time, as well as aim, if applicable. Together the GMP and parameter settings form a motor response action plan. After the action plan is carried out, four things are temporarily stored in working memory: the initial conditions, response specifications, response outcome, and sensory feedback. By extracting constructive information from each attempt, the relationship between parameter selection and the intended outcome is enhanced (Schmidt, 1975; 
1988; Van Rossum, 1990). Hence, practice utilizing a variety of parameters will increase the potential for response accuracy in any context, novel or experienced. If the Variability of Practice Hypothesis is correct as stated by Schmidt, then more variable practice will result in motor response schema refinement. As a result, greater efficacy of appropriate parameter selection for a specific outcome occurs and skill performance improves.

Variability of Practice has been examined extensively in numerous settings involving cognitively controlled motor skills. Strong support for the effectiveness of variable practice has been demonstrated in many different studies examining sports related skills (Goodwin, Eckerson, Grimes, and Gordon,1998; Haudum, Birklbauer, Josef, and Muller, 2011; Memmert, 2006) and simple movements of the hand (Kantak, Sullivan, Fisher, Knowlton, and Winstein, 2011; Lai, Shea, Wulf, and Wright's, 2000; Ranganathan and Newell, 2010). Shea and Kohl (1990; 1991) along with Whitacre and Shea (2000) examined the application of variable practice to prescribed force production. Although the force muscles are capable of producing is not cognitively controlled, the task was to reproduce a force amplitude pattern using a dynamometer, therefore introducing a cognitive component.

The aspect of cognitive processing in the execution of motor skills is a key attribute of the Variability of Practice Hypothesis and Schmidt's Schema Theory. Despite this, the concept of variable practice is often referenced in the strength training field without acknowledging the incongruence of controlling mechanisms. Although an element of central control is supported by mental imaging studies, (Gabriel, Kamen, \& Frost, 2006) significant neuromuscular adaptations and strength improvements originate at the cortical and neuromechanical level (Enoka, 2002; Gabriel et al., 2006). For instance, Pavel 
Tsatsouline, a Russian Master of Sport, and Dan John, a renowned strength coach (John \& Tsatsouline, 2011; Tsatsouline, 2006), propose variable practice as a superior method of strength training compared to a traditionally periodized approach. John and Tsatsouline (2011) suggest that by changing the number of sets, repetitions, and weight used each set "your nervous system will estimate the required force from the stored schema and run the [skill's] motor program with the [specific weight] parameter value" (p. 201). Here the use of the terms "schema" and "motor program" insinuate a direct application of Schmidt's Schema Theory; even though this interpretation clearly differs from motor pattern adaption described in Schmidt's original theories $(1975 ; 1988)$. Furthermore, this diverges from the conventional format of weight training in which the same weight and a fixed number of repetitions for every set is used in a training session, i.e., 3 sets of 5 repetitions of 20kgs (American College of Sports Medicine, 2010; Heyward, 2010). While Schmidt's description of Variability of Practice does not conceptually include strength adaptations, recent strength training research supports the idea that Variability of Practice may be more generalizable than originally proposed to the area of strength training.

\section{Strength Adaptation Facilitation}

Movement is produced by muscular contractions in response to a perceived need; these signals are initiated and controlled by the cortical motor centers (Enoka, 2006). The amount of force muscles can produce is dependent on many different factors, all of which stem from either motor unit recruitment or activation (potential discharge) rate. As a muscle exerts force, motor units are recruited. Force output will continue to increase as long as recruitment and firing rate increase, however, force production past the upper 
limit of recruitment is possible only if firing rate increases. Once recruited, motor units stay active until force output can no longer be sustained; deactivation will then occur in the reverse order. (Enoka, 2002). Adaptability of the CNS to specific prescribed forces is described as the ability to predict and match the muscular force required to produce a desired movement or outcome. Enoka (2006) elaborates explaining that when an internal model is overloaded, changes in length of the muscles involved "evoke long-latency reflex responses" (p. 296). For instance, when a motor program and the structures involved encounter more resistance than previously experienced, a combination of stretch reflex induced and voluntary motor unit recruitment occurs in attempts to achieve the intended outcome (Enoka, 2006; Gabriel et al., 2006). Such alterations can, with sufficient exposure, adapt and change the relative muscle activation to accommodate the new force requirements. Adaptations in response to training will continue resulting in strength improvements as long as stimulation provided by the program is increased in such a way that the neuromuscular system is being challenged (Bird, Tarpenning, \& Marino, 2005).

Periodization is the systematic alteration of training program variables created to avoid performance plateaus, decrease training injuries, and reduce the risk of overtraining for year-round and multi-sport athletes. Proper manipulation of volume (amount of weight lifted) and intensity optimizes neuromuscular stimulation throughout a training program, allowing an athlete to peak at a specific time for competition. If the same stress is experienced for too long of a period the body enters a state of exhaustion and positive changes cease. At this point training schematics must be altered as to expose the body to new stimulation. As the body adapts to handle greater loads, greater stimulation is then 
needed to elicit further adaptations to continue experiencing strength gains (Herodek, Simonovic, \& Rakovic, 2012). Classic linear periodization has been proven effective in strength literature (Bird et al., 2006) to incur neuromuscular adaptations such as motor unit recruitment efficiency and synchronization, increased firing rate, and inter-muscular coordination (Cormie, McGuigan, \& Newton, 2011, Gabriel et al., 2006). Research in the last decade however, has been investigating ways to arrange program schematics in hopes of enhancing strength adaptations resulting in greater strength gains than linear periodization.

\section{Variability within Strength Training}

Strength and conditioning research has recently begun investigating a model of resistance training similar to Pavel Tsatsouline and Dan John's recommendation known as undulating periodization (Miranda, Simao, Rhea, Bunker, Prestes, Leite, Miranda, Frietas de Salles, \& Novaes, 2011; Monteiro, Aoki, Evangelista, Alveno, Monteiro, Picarro, and Ugrinowitsch, 2009; Rhea, Ball, Phillips, and Burkett, 2002; Simäo, Spineti, Freitas de Salles, Matta, Fernandes, Fleck, Rhea, \& Strom-Olsen, 2012). Undulating periodization (UP) involves alterations in volume and intensity more frequently than linear periodization. Another form of UP is daily undulating periodization (DUP) in which volume and intensity is different every training day of the week. Rhea et al. (2002) for example, utilized DUP consisting of an intensity and volume on day one of 3 sets at subject's 8 repetition maximum (8RM), meaning a load that can only be lifted for 8 repetitions, 3 sets of $6 \mathrm{RM}$ on day two, and 3 sets of $4 \mathrm{RM}$ on day three every week. John and Tsatsouline's (2011) model could be described as session undulating periodization, as the load and number of repetitions vary every set, every lifting session. Such a program 
design introduces variability by waving intensity and volume from set to set. For instance the first set might be at $80 \% 1 \mathrm{RM}$ for 5 repetitions, then one at $65 \% 1 \mathrm{RM}$ for 7 , another at $85 \% 1 \mathrm{RM}$ for 3 , and one at $90 \% 1 \mathrm{RM}$ for 2 repetitions. This back and forth alteration of intensity and volume is indicative of an undulated periodization.

Conversely, linear periodization (LP) is characterized by gradual decreases in volume and increases in intensity over longer periods of time, most commonly every 4 weeks for 8 to 12 weeks total (ACSM, 2010; Heyward, 2010). Typically intensity will begin at moderate levels such as $60 \%$ to $70 \% 1 \mathrm{RM}$ while volume is higher, i.e. 3 to 4 sets of 8 to 10 repetitions. Usually after about 4 weeks, intensity is increased to anywhere from $75 \%$ to $85 \% 1 \mathrm{RM}$ and volume decreased to 5 to 8 repetitions. This progression continues for the duration of the program and varies depending on the length of the program, training goals, and types of lifts emphasized. To summarize, training programs that use UP implement greater variation than the classical LP models. The main difference in periodization designs is the frequency and direction of change in volume and intensity throughout the program. While all three periodization styles are effective, UP and DUP programs have been shown to produce substantially greater strength gains (Monteiro et al., 2009; Rhea et al., 2002; Siamo et al., 2012). Although this is a not a direct application of variable practice as described by Schmidt $(1975 ; 1988)$ or Tsatsouline and John (2011), these findings support the concept that increased variability during strength training could be an influential factor in the neurological adaptations involved in the strength improvements. These studies prompt the need for further research to examine the validity of these findings as well as which program elements, when varied, augment neuromuscular adaptations. While a direct statement of variable 
practice's applicability to weight lifting and neuromechanical adaptations is not definitive, the existing empirical evidence is favorable.

\section{Perception of Exertion}

There are many claims associated with strength training research that certain programming designs allow for greater recovery or reduced stress on the muscles. The waving of intensity and volume as described by Pavel and Dan John (2011) is suggested to permit more muscle recovery due to the presence of lower intensity sets allowing more recovery before sets of higher intensity. Current research examining increased variation in strength training supports this statement proposing frequent changes in stimuli places greater stress on the neurological components of the neuromuscular system than the muscles themselves. Hence, it is believed the neurological stress is what forces the system to make further adaptations without substantial morphological contributions (Miranda et al., 2011; Monteiro et al., 2009; Rhea et al., 2002; Siamo et al., 2012). While there are studies that have validated the use of a ratings of perceived exertion (RPE) scale specific to weight lifting, (Day, McGuigan, Brice \& Foster, 2004) RPE has yet to be measured in research examining different strength training formats.

\section{Statement of Purpose}

The primary purpose of this study was to examine whether Variability of Practice enhances neuromuscular adaptations when compared to traditional strength training. Theoretically, by implementing variable practice during strength training, the motor units are exposed to different force settings (Enoka, 2006). A different weight means a new force parameter, which if varied during practice results in refinement of the motor 
response schema. As a result, when lifting a novel or in this particular case a heavier weight, the neuromuscular system can program the appropriate response to successfully complete the range of motion of the lift. If variable practice does indeed facilitate greater neuromuscular adaptations by the mechanisms described, then the variable practice group should outperform the traditional group as seen by greater strength gains after training.

The secondary purpose was to investigate whether there was a difference in perception of exertion between the two strength programs. John and Tsatsouline (2011) propose that increasing variability within a lifting regimen decreases physical strain. Recent strength research claims that the more frequent stimulus changes in DUP result in a superior stress to recovery ratio when compared to LP (Miranda et al., 2011\& Simäo et.al, 2012). If variable practice as described by Tsatsouline and John (2011) is a more efficient method of achieving adaptations than traditional training models, then the variable practice group should report lower RPE values than the traditional group.

\section{Delimitations}

The study was delimited to the following core parameters: All subjects were recreationally experienced weight lifters between the ages of 18 and 45, and recruited from the central coast of California. Additionally, results and findings are specific to the kettlebell press and leg press trained via the two periodized programs examined in the present study. 


\section{Assumptions}

It was assumed that experimental and control subjects adhered to agreed participation requirements. It was also assumed that subjects in training groups gave $100 \%$ effort for every lift during every session.

\section{Hypotheses}

It was hypothesized that the variable treatment would attain greater strength gains than the traditional treatment as measured by the difference between 1RM measures post 8 weeks and baseline. The basis for this relates to previous research demonstrating variable practice as superior for enhancing parameterization of a skill (Goodwin et al., 1998; Haudum et al., 2011; Kantak et al., 2011; Lai et al., 2000; Memmert, 2006; Ranganathan \& Newell, 2010; Shea \& Kohl, 1990; Shea \& Kohl, 1991; Whitacre \& Shea, 2000). Emerging strength training sources also advocate for increased variability within strength training programs, claiming greater neuromuscular adaptations are induced compared to traditional formats (John \& Tsatsouline, 2011; Miranda et al., 2011; Monteiro et al., 2009; Rhea et al., 2002; Simäo et.al, 2012).

Recent research on strength training also supports our secondary hypothesis that the subjects undergoing the variable treatment would perceive lifting bouts as less strenuous than those undergoing the traditional treatment. This may be possible due to a greater stress to recovery ratio (John \& Tsatsouline, 2011; Miranda et al., 2011; Simäo et.al, 2012) displayed by lower reported RPE values. 


\section{Definition of Terms}

Variability of Practice Hypothesis: Practicing more variations of a task, that is practicing many different parameter settings, will facilitate learning of a new motor pattern, resulting in greater potential to successfully execute that task (Schmidt, 1988).

Variable Practice: Systematic variation of a task by changing a parameter within the same GMP (Hall, 1990; Schmidt, 1975; Schmidt, 2003; Schmidt \& Lee, 2005).

Generalized Motor Pattern (GMP): An abstract central representation of a motor pattern that governs a class of actions. This motor pattern is defined by its invariant features: relative timing, force, and movement sequence (Hall, 1990; Schmidt, 1988).

Invariant Features: Characteristics of a movement which remain consistent with each variation. These are constant regardless of the parameter setting, i.e., order or sequence of movement components, relative timing, or relative force (Schmidt, 1975).

Class of Actions: A closely related group of movements defined by the invariant characteristics they have in common (Schmidt, 1975).

Parameter: A component of a motor skill that must be specified and programmed into a GMP based on the goal of the movement outcome, i.e., limb selection, absolute force, absolute timing, or aim (Schmidt, 1975).

Motor Response Schema: The relationship of a specific parameter setting to the intended movement outcome (Schmidt, 1975; 1988). 
Kettlebell: A Russian free weight that looks like a cannon ball with a handle on the top. The handle sits inside the hand with the thumb wrapped around making a neutral grip while the bulk of the weight sits on the dorsal side of the wrist (Tsatsouline, 2006).

Kettlebell Press: A combination of the dumbbell shoulder press and the Arnold press, but with a kettlebell. From a standing position, the hips and knees remain extended throughout the press and the shoulders stay square. This ensures the spine stays neutral, without hyperextension or lateral deviation. The weight distribution of a kettlebell differs from a dumbbell requiring a modified initial position compared to a standard dumbbell shoulder press. The kettlebell handle is held across the palm with a closed grip with the mass of the bell on the dorsal side of the wrist. The ipsilateral elbow is tucked in, sitting on the ribs, and the forearm and wrist are vertical with the knuckles directed at the sky. Concentric movement is upward and slightly outward from the anterior axillary line of the torso, with a slight internal rotation of the forearm until the entire arm is vertical. In the final position the elbow is in extension with a neutral wrist and the palm facing forward. Eccentric movement follows this pattern in reverse (Tsatsouline, 2006).

Training Volume: The total amount of weight lifted during the workout calculated by summing the product of the weight lifted, number of repetitions, and number of sets for each exercise.

Training Intensity: Expressed as a percentage of an individual's 1-repetition maximum (1RM).

1-Repetition Maximum (1RM): The maximum weight that an individual can lift for 1 complete repetition of an exercise. 
Periodization: A systematic variation of intensity and volume in a resistance training program by manipulating one or more training elements, i.e., number of sets and repetitions, percentage of 1RM, or training frequency (Heyward, 2010).

Linear Periodization (LP): A resistance training model that progressively increases intensity as training volume is decreased in cycles of varying lengths (Heyward, 2010).

Undulation Periodization (UP): A resistance training model in which training volume and intensity can increase and/or decrease biweekly, weekly, or daily (Heyward, 2010).

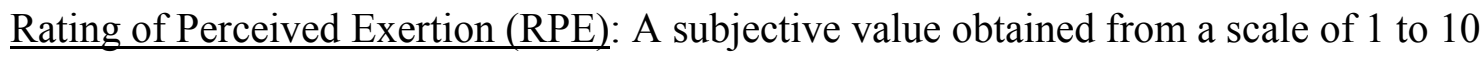
individuals can use to rate their degree of exertion or effort during exercise (Heyward, 2010).

Intermediate Lifters: Habitually resistance-trained persons with previous weight lifting instruction, while not being competitive weight lifters or collegiate athletes with structured strength training regimens (i.e., football, baseball, or wrestling). 


\section{CHAPTER II}

\section{Literature Review}

\section{Introduction}

Effective Variability of Practice studies have at least two experimental groups, a variable practice group and a constant practice group. While constant practice $(\mathrm{CP})$ is of only one task variation at a time, variable practice (VP) includes multiple variations of a task concurrently. Some research designs also include a control group for comparison to verify effects are a result of the assigned treatment. Several variables controlled by the researcher can affect the outcome of the study including sufficient practice time and use of appropriate tests to assess learning. Adequate acquisition time in the form of multiple days or trial blocks is needed to ensure learning can take place within the study design. Learning must then be assessed using retention or transfer tests. Retention tests must be tasks that all treatment groups had time to practice and follow completion of the acquisition phase. Typically, retention tests are administered after a time period of no practice following acquisition, to test levels of learning. Some studies will do both an immediate retention test as well as a delayed retention test 24 or 48 hours after acquisition. Transfer tests, on the other hand, are task variations not practiced by any group, therefore testing ability to set appropriate parameters to a GMP in a novel context. Not all variable practice studies include a transfer test and just use retention test(s) to determine overall learning levels. To test transferability of learning to unpracticed task variations, a transfer test is needed. 
Typical resistance training (RT) programs for strength involve a fixed number of sets, repetitions, and intensity defined by a percent of the individual's 1-repetition max (1RM). For strength improvements, 2 to 4 sets are usually performed with no more than 8 repetitions per set. Rest intervals of at least a minute and as long as 3 minutes, are needed to ensure successful execution of the next set (ACSM, 2010 \& Heyward, 2010). Generally, linear periodization is implemented, gradually decreasing volume and increasing intensity throughout the resistance training program. While this format is effective, more recently the application of "variable practice" has been utilized in strength training by way of undulating periodization. This format includes more variability of volume and intensity which is said to provide greater stimulation for the neuromuscular system. For this reason, UP formats for strength training are accepted by many professional coaches as being most effective method of inducing strength gains, while reducing the risk of overtraining and overuse injuries (John \& Tsatsouline, 2011).

In this research review, Variability of Practice studies that follow the effective format previously described will be referred to as model studies. Generally, model studies have provided support for the theoretical principles of the area. While there are many effective treatment designs, confounded studies occur when one or more practice treatments are not indicative of Variability of Practice principles. Interpretation of results is then unclear as to which theory's mechanisms are responsible. Additionally, study designs in which context effects are present, invalidate results presented during examination of Variability of Practice. Research in the area of strength training is currently investigating the effectiveness of classic linear periodization compared to undulating periodization which some consider a form of variable practice. While many 
studies provide evidence of undulating as superior over linear periodization, there are also studies that equate the two method's effectiveness.

\section{Variability of Practice Research}

Model Research. Variable practice methodology has been applied to many different types of tasks. A classic study investigated timed arm movements across different distances using a barrier knockdown apparatus (McCracken \& Stelmach, 1977). Subjects were randomly assigned to one of three experimental conditions: High-instance (HI), which constituted as variable practice, low-instance (LI) as constant practice, and a control group. HI practiced 4 different horizontal distances, 15, 35, 60 and $65 \mathrm{~cm}$, with a target time of $200 \mathrm{~ms}$ for a total of 300 trials ( 75 of each) in random order. LI practiced 1 of the 4 distances, assigned to subjects in a rotational manner, for a total of 300 trials. The control group practice was set up the same way the LI group; however they only performed 75 trials of their assigned distance. After acquisition all three practice groups performed 30 trials on a new distance of $50 \mathrm{~cm}$ (immediate transfer test). Twenty-four hours after acquisition subjects returned for another 30 trials of the same transfer distance (delayed transfer test). Absolute error (AE), constant error (CE), and variability error (VE) were measured and analyzed for transfer blocks to assess transfer of learning to a novel version of the task. The control group had minimal practice and was used as a comparison group, similar to setting a baseline. HI and LI groups were further analyzed to determine a difference due to variability between only $\mathrm{HI}$ and LI. AE revealed significant differences for the immediate transfer test with HI having lower errors; however differences were not significant after 24 hours. Findings support schema theory in that variable practice resulted in greater transfer performance to an unpracticed task 
parameter, however, effects did not last 24 hours. Since performance of practiced parameters was not better than groups without variable practice conditions, schema theory was not supported as greater overall learning levels did not occur between variable practice and constant practice groups.

A well known study in the area of Variability of Practice was conducted by Shea and Kohl (1990). As a comparison between the practice specificity principle and Variability of Practice hypothesis, subjects were randomly assigned to one of two groups, specific practice (which is equivalent to $\mathrm{CP}$ but will be denoted here as $\mathrm{S}$ ) and variable + specific practice (which is variable practice, but will be denoted here as SV). The task was to produce a target force by applying force with a single arm to a handle attached to a force transducer with their elbow at $90^{\circ}$. Immediate feedback was given via computer screen with a graph showing their force magnitude versus the prescribed force so they could gauge their next trial. The $\mathrm{S}$ group performed 17 acquisition blocks of 5 trials each, attempts spaced every 16 seconds resulting in 85 total trials at $175 \mathrm{~N}$ (criterion force). The SV group performed 17 acquisition blocks of 17 trials, 5 being at criterion force, with attempts spaced every 4 seconds. Every 4 th attempt was at criterion force while 3 variable targets, 25 and 50 Newtons above and below the criterion force were performed in between. This resulted in 289 total trials, 85 of which were at the criterion force. Two retention tests of 5 attempts each at criterion force were administered 24 hours after acquisition presented with 16 seconds between trials. Findings indicated that variable practice made significantly lower absolute errors than the specific practice group. Conclusions from experiment one include that changing the time frame between trials improved motor response relative to massed practice, and variable practice interspersed 
between specific practice resulted in a more robust schema, allowing more accurate parameter selection.

To compare SV effects relative to S with equal practice trials Shea and Khol conducted a follow up experiment in the same publication. The protocol was replicated with the exception of an additional group (specific-specific, denoted here as SS) that performed the same number of trials as the SV group, done in 17 blocks of 17 trials, attempts spaced every 4 seconds, all at the criterion force. The retention test was administered 24 hours after acquisition and consisted of two blocks, 5 trials each, an attempt every 16 seconds (same as first experiment). Even with number of trials matched, SV group performed significantly better than both specific practice groups (S \& SS) supporting the notion that variable practice facilitates retention of a task to a greater extent than specific practice. Shea and Kohl theorize that only specific practice may have resulted in relying on augmented feedback to respond appropriately in the following trial. Meanwhile combination variable and specific practice can do this from memory. It is also important to note that when variable practice was presented randomly, although it increases errors during acquisition, it resulted in fewer errors in retention.

Shea and Kohl (1991) continued their research further examining variable practice learning effects using the same task. Subjects in this study were randomly assigned to one of four groups, all of which did 20 blocks of 5 trials each at $150 \mathrm{~N}$. The specific-spaced group (S) had attempts spaced every 16 seconds within blocks where as the specificspecific group had 3 additional attempts at the same force prescription resulting in attempts every 4 seconds and a total of 17 trials in each block. The specific-variable group (SV) had the same timing as the SS group, but performed 3 different force targets 
( 25 and $50 \mathrm{~N}$ above and below $150 \mathrm{~N}$ ) between criterion attempts. A fourth group followed the same practice schedule as the SS group, however between attempts they performed an unrelated force tracking task using a hand dynamometer with the opposite hand (SU). Shea and Kohl suggested that depending on which group performed superiorly the locus of benefit for variable practice could be identified. They hypothesized that if the SV group showed greater retention then the elaboration perspective explained advantages where as is if the reconstruction hypothesis was the primary mechanism, the SU and SV would have similar retention levels. Results indicated that SV had significantly lower variable errors as well as constant errors compared to all other groups. SS had the most variable and constant errors followed by $\mathrm{S}$ and SU which did not differ significantly. Two theories used to explain possible mechanisms for which retention is enhanced from forms of variable practice are the elaborative perspective and Lee and Magill's reconstruction hypothesis. The elaborative perspective had been described as prior experiences of a task variation in working memory during processing of a current task variation. Elaborative or distinctive processing occurs allowing the individual to recognize the different details of each variation, resulting in greater ability to perform specific task outcomes depending on the goal. The proposed reconstruction hypothesis states that when a learner performs a task, the action plan for a previous task must be abandoned and a new one constructed for the present one. Being required to reconstruct action plans for each new task increases the ability to construct and execute new action plans, allowing for greater performance of new tasks or task variations. Findings of this study counter general notions of practice as all conditions with less practice of the criterion force performed better on retention with lower errors and variability which supports the elaborative perspective. The combination 
of specific and variable practice (SV) provided multiple related tasks in working memory, facilitating distinctive processing and enhanced retention. Finding did not support the reconstructive theory as practice designed to promote forgetting and reconstructive processing performed inferiorly on retention. In addition, practice including unrelated tasks interspersed between specific practice and or increased time between specific practice trials showed no benefits in retention performance.

Experiment 2 was conducted to further investigate the elaborative perspective by testing how many interim tasks promote the greatest retention. Totaling 6 experimental groups, specific and specific-variable (SV) practice include either 0,1 , or 3 different task variations between criterion attempts during acquisition. Interval times of each attempt were also varied as either every 10 or 30 seconds. For groups with 1 and 3 different force prescriptions ( 25 and $50 \mathrm{~N}$ above and below criterion again) the order of presentation was random. The task was also changed to a dynamic force production rather than static, so subjects hit a force transducer at a given time to produce a prescribed force. Subjects sat at a table with a computer displaying a horizontal line as baseline and a line above which marked the prescribed force; when they hit the transducer, their applied force was shown as a vertical line steaming from baseline up to the prescribed force. Specific practice with 10 seconds between trials (S-10) completed 17 blocks of 5 trials totaling 85 . The S-30 group did the same thing, but with attempts every 30 seconds. Specific-variable practice with 1 additional task variation and 10 seconds between attempts (SV1-10) completed 17 blocks of 9 trials ( 5 of criterion, with 1 of the variable forces between each criterion attempt) while the SV1-30 group's attempts were 30 seconds apart. The SV3-10 group preformed 17 blocks of 17 trial attempts ( 5 of criterion with 3 variable force attempts 
between) and the SV3-30 group's attempts being spaced every 30 seconds. The SV1 and SV groups had 152 and 289 total trials respectively. Retention was measured 24 hours after acquisition and consisted of 5 trials at $150 \mathrm{~N}$ with attempts spaced every 10 seconds in a repetitious manner. Statistical analyses revealed lower constant absolute errors in SV1 and SV compared to specific practice, but did not differ from each other. The only significant finding regarding different trial timings was that specific practice groups had significantly lower constant and absolute errors with attempts every 30 seconds compared to every 10 seconds. While the elaboration theory does not specify the number of items needed in working memory for optimal learning effects, results support the elaborative perspective as practice with increased variability performed the best on retention. It was also suggested that increasing the time between repetitions could be beneficial, as too short of intervals might hinder processing and acquisition of the skill. Findings suggest that with sufficient time between attempts of a skill, increased variability of practice will improve learning of a skill.

Another research endeavor using the same type of apparatus was done by Shea and Whitacre (2000). Once again this research was conducted using two experiments. The first involved variable practice in every group as a means to assess how variable manipulation effects both GMP and parameter learning. Two tasks had 3 variations each in which subjects tried to produce force amplitudes matching a pattern displayed in waveform. Relative force and timing as well as absolute timing were kept constant for all versions of each task (A1, A3, A5, B1, B3, \& B5) with varying absolute force targets. Subjects were randomly assigned to one of four groups, two of which performed all task A waveforms, differentiated by number of acquisition trials. One group only completed 2 
blocks of 10 trials each while the other completed 10 blocks of 10 trials. Both groups had absolute force being varied from trial to trial to investigate parameter learning. The other two groups followed the same format for task B waveforms. Five minutes after completion of acquisition, a retention test of 10 trials of waveform A3 for group A and B3 for group B was administered. A delayed retention test was administered in the same manner 24 hours after acquisition. Analysis revealed RSME errors, which encompass GMP and parameter errors, were lower in groups who had 200 vs. 20 trials worth of practice, but increased for all groups from immediate to delayed retention tests. RSME residual errors, exhibiting GMP errors, were also lower in groups with 200 trials of practices as well as lower in groups who performed task A rather than B. Period errors, expressed errors in timing of the force parameter which were greater during the delayed retention test as opposed to the immediate. Force errors, force production parameterization errors, were lower in those with 200 trials of practice compared to 20 trials as well. Task almost had a significant effect as those in group B had more force errors than those who did task A. Deterioration in total RSME from immediate to delayed retention tests as compared to residual RSME was much greater, thus the majority of errors seen from immediate to delayed retention is believed to be due to degradation in parameter learning. In addition, the GMP for task A was also shown to have been more developed than task B, which Shea and Morgan hypothesized to be because it had a simpler GMP. It is also important to note that variable practice only enhanced learning of the manipulated variable (absolute force), whereas the non-varied parameter (absolute timing) was unaffected. 
The purpose of experiment 2 (Shea \& Whitacre, 2000) was to examine variable practice more directly. The first experiment theoretically allows examination of the task and practice variability's interactive effects on GMP learning versus parameter learning. The apparatus and tasks were the same in experiment 2; however, each task now had 5 versions, each differing in prescribed magnitude. Subjects were randomly assigned to one of four groups defined by either task A or B as well as either constant or variable practice (AC, AV, BC, BV). Constant practice groups performed the either $\mathrm{A} 3$ or $\mathrm{B} 3$ while variable groups were presented with a different version of A or B every trial in random order with the exception that all versions were displayed an equal number of times. Acquisition for all groups included 20 blocks of 10 trials (200 total trials) with retention tests of task A3 and B3 after 5 minutes and 24 hours of acquisition completion. RSME (total errors), residual RSME (GMP errors), force (varied parameter) errors and period (non-varied parameter) errors were all measured again. Total RSME results exhibited lower combined GMP and parameter errors for those in constant practice groups, however, errors increased from immediate to delayed retention tests. The only significant residual RSME result, representing GMP errors, was that fewer errors occurred in groups performing task A than those performing task B. Constant practice groups experienced lower timing parameter errors and more stability from immediate to delayed retention tests whereas variable group's errors increased from immediate to delayed retention tests. Force parameter errors were not different across practice types or tasks; this is an important finding as constant practice groups had significantly more practice trials on A3 or B3 compared to variable groups (all 200 trials vs. 40). Analysis suggests that learning was greater in the constant practice groups shown by less decay from immediate to 
delayed retention tests when compared to variable practice. Findings also indicate however, that variation of one parameter caused poorer learning of the parameters not varied.

Benefits of variable practice have been demonstrated in many studies; examination of how much variable practice is advantageous is another avenue of interest. Goodwin, Eckerson, Grimes, and Gordon (1998) utilized two variable practice groups and a specific only practice group to explore how the degree of variability during practice influences learning. Subjects were randomly assigned to either specific practice, specific-variable, or specific-varplus, with the exception of ensuring the total subject number and female to male ratio was the same. The task was dart throwing from different distances from the target with specific only practicing from the criterion distance of $2.39 \mathrm{~m}$ for 75 trials. Specific-variable practice included the criterion distance and two others $(1.47 \& 3.30$ meters) while specific-varplus had an additional 2 distances (1.93 \& 2.84 meters). Both variable groups performed 75 trials in quasi-random order with no more than 2 consecutive trials being the same and equal trials for each distance. Retention and transfer were assessed 24 hours later with 15 trials of the criterion distance and 15 trials of a novel distance of $3.76 \mathrm{~m}$. Retention data analysis revealed no significant differences between groups, however, conditions with supplemental variable and varplus practice did fewer trials of the criterion task than did specific practice only. Transfer results revealed both variable groups performed significantly better than specific practice. These trends suggest specific practice with supplemental variable practice leads to enhanced learning of a skill. Results also support schema theory's prediction of variable practice creating increased transferability of learning effects to new parameters. 
Common tasks to test motor learning theories are simple finger manipulations like Lai, Shea, Wulf, and Wright's (2000) relative time constrained finger depression pattern of 4 fingers with varying absolute timings. Examination of relative and absolute timing allowed them to test notions of constant and blocked practice fostering more stable learning of the invariant features of a GMPs while variable and serial practice resulting in greater parameter learning. Use of knowledge of results (KR) was also investigated in terms of bandwidth KR versus quantitative KR. Bandwidth KR refers to qualitative KR in which feedback is given based on performance within or outside a specified range as opposed to a single target. In this particular study Lai et al. (2000) used a bandwidth of $0 \%$ where quantitative KR was given after every trial regarding by how much their timing was off target. Bandwidth KR of $15 \%$ groups were given qualitative KR of "correct" as long as their performance was no more than $15 \%$ off. If more than this, subjects were given quantitative KR with information as to how far off they were. Subjects were randomly assigned to one of four practice groups: constant practice with $0 \%$ bandwidth KR, constant with $15 \%$ bandwidth $\mathrm{KR}$, variable practice with $0 \%$ bandwidth KR, or variable with $15 \%$ bandwidth KR. During acquisition, each task version's relative timing between key depressions was held constant while absolute timing was varied. Relative and absolute timing for each trial was displayed before execution and KR was presented immediately after task completion. All groups did 9 blocks of 12 trials totaling 108 trials. Constant practice groups only did version B for the entire acquisition phase while variable groups were presented with A, B, and C serially in that order. Retention and transfer were assessed on the following day with 5 minutes between the retention test and transfer test. Retention was a block of 12 trials of task B 
while transfer was an absolute time not practiced in acquisition. As previous research suggests, constant practice resulted in more stability of invariant features while variable practice enhanced transfer to new parameters. This study provides more evidence that GMP and parameter learning are independent of each other and a combination of constant and variable practice is needed for optimal results. In addition, effects of bandwidth KR seemed to benefit variable practice more so than constant groups. Lai et al. (2000) attributed this to constant practice providing sufficient stability and thus less to gain from the external feedback.

Lai et al. (2000) extended this study to examine the best order of constant and variable combination practice. Subjects were divided into four groups comprised of either all constant, all variable, constant-variable, or variable-constant practice. Combination groups completed the first half of acquisition with one type and the second half with the other. The all constant and the constant-variable practice groups resulted in superior performance on retention and transfer tests 24 hours post acquisition completion. These findings are believed to show the greater GMP learning and lasting effects of constant practice early in acquisition. All variable and constant-variable practice groups performed the best on transfer supporting parameter learning enhancement is due to variable practice. Together these results can be used support the idea of combination practice for optimal learning, especially since the constant-variable seemed to experience the greatest overall GMP and parameter learning effects. These findings suggest constant practice should be utilized when first learning a motor task to develop a strong GMP while variable practice should be used later to strengthen schema response. 
While much research has examined task goal variability, Ranganathan and Newell (2010) also investigated effects of variability in strategy of a targeting task. The criterion task involved drawing a line on a digitizing tablet from a static start point, through an obstacle to a static target. To match ability for all groups, after completing a pre-test of 50 trials subjects were assigned to one of 4 groups. Acquisition included 8 blocks of 50 trials where constant practice performed the criterion task and the variable practice group had a target point that shifted vertically by $4 \mathrm{~cm}$ in either direction randomly from trial to trial. These two groups served as a kind of control to compare the two groups designed to examine drawing path variability. Variability in the drawing path was introduced by an intermediate target subjects had to draw through before proceeding to the final target (VPP: variable path practice). This intermediate target shifted $4 \mathrm{~cm}$ vertically in either direction randomly from trial to trial. Constant path practice had the same start point and final target with the intermediate target placed in line with the average path of the criterion task (CPP). An immediate constant practice retention test was completed by all subjects right after acquisition which was comprised of 50 trials of the criterion task. A variable target retention test of 50 trials was also administered to every subject. Twentyfour hours later, both constant practice and variable target retention tests were completed again. Analysis of all groups on performance of the constant practice test revealed no significant differences between any groups. Comparison of just the variable target practice and constant practice groups showed constant practice as having performed with significantly lower absolute errors during immediate, but not during delayed constant target conditions. During the variable target test, delayed performance was better in all groups, but the variable group exhibited significantly lower absolute errors than all other 
practice groups. Findings of Raganathan and Newell bring to light that variability introduced during task execution did not provide advantages to learning, but variability to the target outcome did. However, findings are task specific and might not apply to the notion that learning multiple ways to complete a task has no benefits to GMP learning.

One interesting application by Yao, DeSola, and Bi (2009) examined maintenance of specified wheel chair speeds. There were two constant practice groups, one training to maintain 30\% max speed pushing a wheel chair, the other at $55 \%$ based on an obtained max speed $(100 \%)$. The variable practice group practiced both speeds, half the trials each day consisting of each speed. Before acquisition a pre-test of four different speeds (30\%, $40 \%, 55 \% \& 70 \% \max$ ) was conducted where subjects were asked to estimate and maintain the given speed for 5 minutes. Acquisition consisted of 10 weeks, 3 training sessions a week, for a total of 6 five minute blocks followed by post testing of the same four pre-test speeds (30\% and $55 \%$ as retention tests, $40 \%$ and $70 \%$ as transfer tests). Subjects were randomly assigned one of four post testing orders where absolute errors were used as dependent measures. Results revealed the variable group to have significantly lower error scores than the $30 \%$ practice group for both novel speeds, $40 \%$ and $70 \%$, but only lower compared to the $55 \%$ practice group for the $70 \%$ condition. Findings show that constant practice was not superior to variable practice for learning of the practiced speeds. Additionally, variable practice was significantly better at setting parameters for new speed that hadn't been practiced. Results propose that variable practice is just as effective for learning a skill as constant practice, but enhances transfer ability to parameters unpracticed. Authors also mention that while Schmidt's schema theory was originally meant to be applied to discrete skills, the task used in Yao et al. 
(2009) is a continuous skill. Therefore, findings propose the Variability of Practice hypothesis can be applied to learning continuous skills as well.

The need for real world relevance of motor learning theories is an important aspect of research. Stroke victims often have to relearn to walk or use affected limbs, therefore if there are advantages to a specific practice schedule or composition then it should be implemented in rehabilitation programs. Rhea, Wutzke, and Lewek (2012) recruited subjects who had suffered a stroke resulting in hemiparesis. They aimed to determine if a single session of training would elicit changes in gait dynamics using video based motion analysis. The protocol was a counterbalanced crossover of treadmill walking sessions consisting of constant and variable speed training. The constant speed training (CST) involved walking at the fastest pace the subject could maintain comfortably (100\% CST). Variable speed training (VST) started with the $100 \%$ CST pace for 5 minutes, but then experienced changes in speed every minute to either $60 \%, 80 \%$ or $100 \%$ CST. Each speed was presented 5 times after the first 5 minutes, never consecutively, and ending with $100 \%$ CST. For both training types, gait during minutes 3 and 4 were analyzed. Speed training sessions lasted 20 minutes followed by a 30 minute break. Each session ended with a 5 minute retention test where all subjects walked at 100\% CST with minutes 3 and 4 used for gait analysis. Subjects completed the other practice type 3 to 14 days after the first, followed by the same retention test. No differences were found between practice types in knee or hip angles deducing that one session of constant or variable training is unlikely able to induce significant gait changes.

Kantak, Sullivan, Fisher, Knowlton, and Winstein (2011) contributed an intriguing application of variable practice by investigating areas of the brain responsible for 
memory consolidation of GMP vs. parameter learning. Previous research identifies the primary motor cortex as being responsible for memory consolidation after learning a new task using constant practice. For variable practice conditions, the dorsolateral prefrontal cortex is responsible for memory consolidation. Based on the fact that interference post acquisition can inhibit consolidation and deter retention, the aim of Katak et al. (2011) was to test if this theory applied to transfer of novel variations of a learned task. A criterion tracking task of $60^{\circ}$ wave amplitudes with the non-dominant hand, for 120 trials, was done for constant practice. Variable practice consisted of 4 versions $\left(30^{\circ}, 45^{\circ}, 60^{\circ} \&\right.$ $75^{\circ}$ ) of similar structure and absolute time duration. Task variations were presented randomly with the exception that 60 trials of the $60^{\circ}$ variation and 20 trials each of all the others were performed during acquisition. Two different immediate (IT) and delayed (DT) transfer tests were administered after the retention test and 24 hours post acquisition. Transfer tests included 8 trials of two different novel conditions, one within range $\left(50^{\circ}\right)$ and one outside the range $\left(80^{\circ}\right)$ of versions practiced by the variable group. Retention was assessed before each transfer test using the criterion tracking task for 8 trials. While no significant differences were found in RMSE errors between groups, transfer revealed similar trends as seen in traditional variable practice research supporting variable practice as facilitating greater learning of parameterization even after 24 hours.

To examine the effects of interference on transfer, 3 different constant and variable practice groups each were included. One group of each practice type (CP-M1 and VPM1) received transcranial magnetic stimulation (rTMS) to their primary motor cortex right after the immediate transfer test (IT). In addition, 1 group of each practice type (CPDLPFC and VP-DLPFC) received rTMS to their dorsolateral prefrontal cortex (DLPFC) 
right after IT. The last two groups were controls for constant and variable practice, receiving no rTMS. For the $80^{\circ}$ transfer test, analysis of these extra conditions revealed that rTMS after IT had no affect on delayed transfer (DT) performance for CP-DLPFC compared to the constant control group. The CP-M1 group however, was affected by rTMS after IT, resulting in poorer performance on DT. Opposite findings occurred in variable practice groups for the $80^{\circ}$ transfer condition; VP-M1 saw no differences compared to variable control groups whereas VP-DLPFC's DT was attenuated. On the contrary, there were no significant effects of rTMS or differences in practice types in regards to the $50^{\circ}$ transfer test. It is proposed this was due to $50^{\circ}$ variation being so similar to the criterion task of $60^{\circ}$, which was tested right before DT for retention, erasing any effects of the rTMS. In conclusion, Kantak et al. (2011) postulated there is a heavier reliance on DLPFC for memory consolidation after variable learning which is in line with the theory of active cognitive processing and its importance for transfer performance. M1 on the other hand was concluded to be more essential in memory consolidation for retention of a new skill as well as transfer after constant practice, however it is hypothesized that there is some overlap of M1 and DLPFC's roles.

Confounded Research. Contextual interference (CI) is another theory regarding the effects of different practice composition and scheduling on learning of motor tasks. Although this review will not explore this concept, many studies use both Variability of Practice theory and CI in the same study because both are based on the principle that increased variability during practice results in greater learning. Shea, Lai, Wright, Immink, and Black (2001) used the same key depression task and sequences as in Lai et al. (2000) and examined both Variability of Practice and CI based practice groups. 
Subjects were randomly assigned to one of four practice groups: constant, blocked, serial, or random. Constant practice performed only task B while the blocked group practiced all 3 absolute timings but one at a time in separate blocks. Serial practice presented the 3 versions one after the other in order $(\mathrm{ABCABC})$ throughout each block while during random practice, which is used sometimes synonymously with variable practice, all 3 tasks were presented randomly with the exception that each task was presented the same number of times throughout acquisition. During blocked, serial, and random practice each task made up 33\% of acquisition. Every group did 9 blocks of 12 trials totaling 108 .

Twenty-four hours after acquisition, retention was assessed with 12 trials of task B and a transfer test 5 minutes later of a novel absolute timing longer in duration than any practiced. Relative timing (invariant feature) errors during both retention and transfer increased from least to most errors in the following order: constant, blocked, serial, random. There were significant differences of constant and blocked compared to random. Serial however, was not significantly different from random or blocked, but was compared to constant. Absolute timing (varied parameter) errors on the other hand were not significantly different between groups during retention, while during transfer smaller errors were seen in random and serial with significant differences compared to constant and blocked practice. These findings provide more evidence for the theory that more consistent practice enhances GMP learning where as random or variable learning enhances the ability to scale movement responses depending on the desired outcome more effectively. This however, cannot be specified as resulting from the CI or variable practice as the combination of practice element manipulation creates confounding factors. 
The study reported by Green and Sherwood (2000) is described as an examination of variable practice structure, however, treatments are practice schedule manipulations of variable practice. This involves $\mathrm{CI}$ which then confounds results regarding effects specific to Variability of Practice. Subjects were randomly assigned to either blocked or random practice of 3 variations of an arm movement task. Using the dominant hand, the forearm and upper arm were positioned to be parallel with the ground. The task was to move the arm while holding onto a grip, moving it from $60^{\circ}$ of elbow flexion to $45^{\circ}$ of elbow flexion, and then back. Movement time was measured from initiation, to the moment of reversal back to the starting position. Blocked practice was comprised of 30 trials of each total movement time $(250,375, \& 500 \mathrm{~ms})$ in a counterbalanced order. Random practice trials were arranged so no one movement time was presented consecutively, but still practiced 30 times. After both groups completed 90 trials, an immediate combination retention-transfer test was conducted as well as a delayed retention-transfer test 24 hours later. During testing, half the subjects from each group were randomly assigned to one of two conditions. One group was asked to verbally estimate their movement time after each trial while the other was not. Test movement times were presented in serial order with a novel movement time included $(650 \mathrm{~ms})$ to assess transfer. The sequence of movement times was presented 10 times for a total of 40 trials each. Random practice provided significantly greater temporal accuracy compared to blocked during immediate and delayed tests. Furthermore, subjects who were required to estimate their errors made significantly less errors. Temporal error detection ability was significantly better for the random practice group versus blocked in both immediate and delayed tests as well. Findings point to random variable practice as developing higher 
levels of overall learning compared to blocked variable practice. In addition, results implicate random variable practice facilitates the ability to detect errors to a greater extent which potentially is responsible for the enhanced error correction ability exhibited in this study as well.

Context Effects. Variable practice can also be applied in a physical education setting. Lidor (1995) investigated whether variability in the high jump approach enhanced performance. Fourth and fifth grade Students in a P.E. class were assessed during the first session of 7 using a vertical jump protocol, in which no significant differences were found in jump ability. Acquisition was done over 1 and a half instructional sessions with one prior being familiarization with the task itself. Students were assigned to one of two groups in which constant practice consisted of starting at the same distance from the jump pit and taking the same number of steps before the jump every time. During variable practice students started at various distances away resulting in a different number of steps for each. It is not stated how many trials students performed for either group or how many different distances the variable group practiced or in what order the distances were performed. The fifth session served as a short term retention test where students performed two high jump attempts. The following session students did general physical activity skills and games unrelated to high jump or jumping skills. On the seventh and last session, students performed two more high jump trials serving as a long term retention test. Statistical analysis revealed no differences in performance on the short or long term retention high jump attempts. These results allege that neither variable nor constant practice is advantageous in comparison to the other in terms of learning augmentation. Acquisition was one and a half school P.E. sessions and retention tests 
were only two high jump attempts each. The short time frame of this study does not support the learning of a sports skill or appropriate assessment of learning. Additionally, there was no control of how "constant" or "variable" the different group's trials were. Collectively, these are confounding factors which invalidate reported conclusions.

Heitman, Erdmann, Gurchiek, Kovaleski, and Gilley (1997) is an example of a study claiming to examine a Variability of Practice concept (transfer) while applying a different theory $(\mathrm{CI})$ during treatment. In addition to producing confounding results because of this, transfer is inappropriate assessed. Heitman et al. (1997) used a barrier knock-down task with children with mental disabilities. Subjects were randomly assigned to one of two experimental groups: constant or variable practice. Three different barrier knockdown patterns were learned by both practice groups, however, the schedule of practice differed. The constant practice group actually used what is referred to as blocked practice in CI research, as each pattern was performed for an entire block of 10 trials before learning the next one. The variable practice group also performed 3 blocks of 10 trials however the 3 patterns were presented randomly, which CI research calls random practice. Day one was referred to as the acquisition phase and day two the transfer phase. Day 2 all subjects performed 3 blocks of 10 trials in the same manner as the variable practice group during day 1 . It is uncommon to repeat an entire acquisition protocol for a transfer or retention test. In addition, this design does not really assess transfer for either group as the patterns being tested have been practiced by all subjects. The testing phase is more along the lines of a retention test, but due to VP having done acquisition in the same format as the test a context effect would ensue resulting in superior performance of the variable practice group. Retention and transfer testing is specific to the tasks practiced by 
treatment groups. Retention is a measure of overall learning of a criterion task whereas transfer is a measure of ability to apply the criterion task schema to a novel context. Therefore retention tests need to comprise of the criterion task which needs to have been practiced by all treatment groups. Transfer tests on the other hand should be composed of tasks unpracticed by any group. Statistical analysis found there were no significant differences between practice schedules on day two. This led Heitman et al. (1997) to conclude that blocked practice does not provide any advantages to learning over variable/random practice for children with learning disabilities. However, the short duration of acquisition, confounding variables due to inclusion of CI elements, and context effects during testing, require further research on practice composition for individuals with cognitive learning disabilities.

Another sports application which involved skill development in 5 and 6 year olds was conducted by Harrison and Keane (2007). Horizontal jump distance (broad jump) was used to assess learning from a plyometric training intervention. Subjects attended 2 training sessions for 30 minutes at a time every week for 6 weeks, of either constant practice or variable practice, focused on developing jumping ability. Prior to acquisition, a broad jump pre-test was administered and horizontal distance measured using video based motion analysis. Constant practice consisted of only vertical jump trials while variable practice included 4 different jump types: broad jump, vertical jump, hopping, and leaping. No other details about acquisition were provided in the report. A broad jump post test was administered after the six weeks of training as well as a retention test one week later. There are a few problems with this design, first of which is that the broad jump task is not a retention test for the constant practice group. As the only task practiced 
by the constant group and one of the tasks practiced by the variable group was vertical jump, that should have been the retention test task. Due to this context effect the variable group outperformed the constant practice group significantly, falsely supporting variable practice as a superior practice form of the broad jump. Another issue is that all the jumping tasks performed by the variable group may not have the same GMP, therefore not providing parameterization practice or GMP practice. Design flaws in the methods in addition to the context effect described, invalidates the conclusions of this investigation. It is important to note that this particular study was not published, but was presented at a symposium.

The following study design is an example of an invalid transfer test when considering the GMP of the acquisition task. Matsouka, Trigonis, Simakis, Chavenetidis, and Kioumourjoglou (2010) conducted a study examining overhand throwing tasks in children with intellectual disabilities. Subjects were randomly assigned to either a control, constant practice, or variable practice group. All subjects completed a 10 trial pre-test of throwing a tennis ball at a target from a distance of 5 meters away (criterion task and distance) as well as 10 trials of a basketball overhand throw into a basketball hoop from the same distance (transfer task). The control group did not participate in any practice while constant and variable practice performed 6 blocks of 20 trials of the criterion task. A total of 120 trials over 3 days (40 trials each day) were completed by both groups. Constant practice only performed the criterion task from 5 meters whereas variable practiced from 3, 5, and 7 meters away in a random presentation, but for the same number of trials each. Criterion and transfer post-tests were executed 24 hours after acquisition and again for what Matsouka et al. (2010) referred to as the retention test, 48 
hours after the post-tests and the transfer test 24 hours after the retention test. All tests were comprised of 10 trials of the associated tasks from 5 meters away. Data analysis revealed no significant differences in retention scores between practice types. Significant improvements were found, however, from pre to post criterion testing, during retention, and during transfer testing for the variable group. No significant improvements were found for the constant practice group. Results of this study propose variable practice facilitates better retention and transfer of an overhand throwing task however a basketball throw and a tennis ball throw likely have different GMPs. Regardless, Matsouka et al. (2010) claims there was a positive effect of practicing throwing a tennis ball at a target from various distances on an overhand basketball throw. No significant differences between practice types, however, suggest the GMPs were too different to result in positive transfer of learning between the two overhand throws.

Memmert (2006) applied a variable versus constant practice intervention to basketball shooting. Subjects were college students who had no experience with basketball other than having completed an obligatory basketball fundamentals course. Constant practice consisted of shots only from the free throw line while variable practice took shots from different distances in and around a defined area. Both practice types used a standard women's size ball ( $72 \mathrm{~cm}$ diameter) and had to use their non-dominant hand. A pre-test of 20 free throws was performed to establish a baseline score before subject assignment to ensure similar skill levels between groups. Acquisition only consisted of 160 trials in one 90 minute session with a break after 45 minutes. Locations of variable practice shots were described as 4 different areas with central points of 3.6 and 4.2 meters to the left and right of the basketball hoop. Ten of the 160 trials were taken from outside of the 
specified region, however, no further specification was given for those locations.

Performance order of shot locations was also not provided, but it stated that order was counterbalanced. An immediate post-test was completed immediately after acquisition and again after 1 year, serving as immediate and delayed retention tests, respectively. Transfer was assessed after the post-test which consisted of 20 trials from 1 meter in front of the free throw line with a smaller ball $(60 \mathrm{~cm}$ diameter) and again 1 year later after the delayed retention test. Qualitative scoring was based on a 0 to 4 point scale: 0 for not even hitting the hoop, 1 for hitting the hoop once, 2 for hitting the hoop twice or more, 3 for making it in the hoop with any hoop contact, and 4 for making it without hoop contact (swoosh). Constant practice performed significantly better on the immediate retention test than variable practice, but variable practice did better than constant practice on the retention test a year later. Both groups saw improvements from immediate to delayed transfer with no significant differences between the two practice types. Previous research is supported as this study displays constant practice as resulting in greater immediate retention, but variable practice facilitating deeper learning exhibited by greater delayed retention. Based on transfer testing, it would seem that variable practice has no advantages compared to constant practice in terms increased parameterization ability of basketball shooting. Then again, this particular study lacks details of the methods that could have contributed to there being no transfer effects. In addition, during transfer two different parameters were manipulated, distance from the hoop and size of the ball. Not only were there no practice conditions related to ball size, most research studies only examine one parameter at a time. 
Another field application was executed using two separate populations, youth basketball and elite volleyball players. Haudum, Birklbauer, Josef, and Muller (2011) conducted two separate protocols for the two populations. Experiment 1 (basketball) started with a pre-test to match groups for skill using a successful to missed shot ratio. Subjects were then assigned randomly to either a control, constant, or differential practice group, with the exception of controlling for skill level. Both experimental groups attended 15 sessions of 50 free throws for seven and a half weeks on top of their normal team practice. Constant practice received no feedback while the differential group's practice focused on a different aspect of performance each session such as knee flexion, knee extension velocity, and wrist flexion during a free throw. No tasks were actually defined or a practice order described for variable practice. The post-test consisted of 20 free throws and measures of their make to miss ratio were recorded as well using the 0 to 4 point system used in Memmert (2006). A retention test is mentioned, but no details of how or when it was administered are provided. In addition a transfer test was conducted using a jump shot task from $45^{\circ}$ to the right of the basket. Twenty trials were performed, all with 3 dribbles before taking the shot. Although differential practice displayed better transfer performance, no significant differences were found in retention or transfer scores among practice types, including the control group. The questionable interpretation and implementation of what they considered variable practice (differential group) and the study taking place in addition to normal team training, results can be attributed to a context effect.

Experiment 2 (volleyball) consisted of 18 sessions, meeting twice a week and completing 25 trials of the volleyball strike per session. All subjects were active national 
volleyball league players and participated in the study concurrently with their normal practices. Subjects were assigned to one of two experimental groups, constant or differential. A pre-test with a constant practice condition and a variable practice condition was administered prior to acquisition. In the constant condition subjects had to strike a ball from the front left side of the net to a specified target in the back left corner as fast as they could for 10 trials. In the variable condition subjects had to spike the ball from the same location 4 times to 4 different target locations (front left, front right, back left, and back right) as fast as they could. Differential practice in this experiment involved external perturbations to the subjects striking pattern by way of resistive cords. Elastic cords were attached to a belt around the subject's waist and connected different anatomical points together such as opposite hip and upper arm or forearm and lower back to upper arm. Further variability was induced by changing the length of the cords. Seventeen of the 25 trials were done with the resistive apparatus, but no more description was given to how many trials of each cord arrangement or locations of the cords were provided. Strikes done without the apparatus were done in the same manner as constant practice, with no feedback and without any interference. Both groups completed post-tests for both the constant and variable conditions following acquisition. Significant differences were found in terms of velocity for the differential group, but there were no differences in terms of accuracy for either test condition. It is likely that because all subjects were elite volleyball players, they were all already extremely accurate resulting in no significant differences. It is also possible, like in the basketball experiment, that because the subjects were participating in regular practice simultaneously, any effects from experimental training protocols are being erased. Like the basketball experiment, the application of 
variable practice is questionable as the varied aspect of the task was movement constraints. Therefore, findings and resulting conclusions may not apply to Variability of Practice effects.

\section{Strength Training Periodization}

Undulated Over Linear. Rhea, Ball, Phillips, and Burkett (2002) were the first to compare linear periodization (LP) and daily undulating periodization (DUP) while matching volume lifted by both groups. Periodization refers to systematically varying the amount of weight lifted and the intensity of weight lifting sessions. LP involves gradual and progressive changes whereas DUP introduces more variability experienced in terms of rate and frequency of these changes. An experience inclusion criterion was at least 2 years of participating in periodized resistance programs. Subjects were randomly assigned to either LP or DUP for a 12 week strength program. Familiarization of both bench press and leg press took place over 6 sessions and 1RM was measured on 3 separate occasions with at least 3 days in between each to establish reliability. There were no significant differences found at baseline between groups. After preliminary testing, subjects trained for approximately 40 minutes each session, 3 times per week with order of bench press and leg press lifting bouts randomized. During weeks 1 through 4, LP subjects completed 3 sets of 8RM, 3 sets of $6 \mathrm{RM}$ weeks 5 through 8 , and 3 sets of 4RM weeks 9 through 12. DUP subjects performed 3 sets of $8 \mathrm{RM}$ on day one of each week, 3 sets of $6 \mathrm{RM}$ on day two, and 3 sets of 4RM on day three for all 12 weeks of the program. All subjects warmed up with a aerobic activity for 10 minutes as well as 10 repetitions of a light weight for each lift. Other lifts included in the program that were not examined were bicep curls, lat pull downs, and abdominal crunches. A minimum of 48 hours 
between sessions and a week of active rest (no weight lifting) between week 5 and 6 was required. While there were no significant differences in body composition within or between groups, both LP and DUP groups experienced significant strength gains in both the leg press and bench press by the end of the program. DUP experienced significantly greater percent strength gains from baseline to week 4 and from baseline to week 12 compared to LP. Significant absolute strength increases were seen in the leg press for LP and DUP after every 4 week period throughout the duration of training. Neither DUP nor LP training resulted in significant absolute strength gains in the bench press at any point. Results in the present study suggest alteration of training variables on a daily basis, as done in DUP, elicits greater strength gains than LP.

It is a well established concept that periodization of any kind is superior than no periodization at increasing strength (Rhea et al., 2002), therefore focus in strength research has shifted to comparing effects of LP versus undulating periodization (UP). Studies such as Monteiro, Aoki, Evangelista, Alveno, Monteiro, Picarro, and Ugrinowitsch (2009) however, used a non-periodized (NP) program as a control treatment. In this study, linear periodization (LP) and non-linear periodization (NLP), which is another term for UP, were compared with NP programming for split training routines. Split routines refer to training alternating muscle groups from day to day, i.e., upper body on Monday, lower body on Tuesday, then upper body again on Thursday, and lower on Friday. Subjects had to be habitually resistance trained for the past 2 years, training 4 days a week. Subjects were randomly assigned to one of the three programs that were equated for volume and lasted 3 months. Each month involved 3 loading weeks and 1 recovery week. Bench press and leg press were assessed using 1RM before 
training, and after week 4, 8 and 12 of training. NP, LP, and NLP programs were divided into sessions A and B. Session A included bench press, incline bench press, decline bench press, lateral raises, military press, triceps pull-down, and the barbell French press. Session B was leg press, hamstring curl, squat, row, lat pull-down, chin up, bicep curl, and the preacher curl. Recovery weeks consisted of bench press, military press, triceps pull-down, leg press, lat pull-down, and biceps curl. During loading weeks all groups trained session A on Mondays and Thursdays and session B on Tuesdays and Fridays. During recovery weeks, sessions were on Mondays, Wednesdays, and Fridays. NP trained all exercises for 3 sets of 8-10RM for the entire duration of training for both loading and recovery weeks. LP did 3 sets of 12-15RM for the first month, 3 sets of 8 $10 \mathrm{RM}$ for the second, and 3 sets of 4-5RM for the third. NLP performed 3 sets of $12-$ $15 \mathrm{RM}$ for the first week of each month, 4 sets of 4-5RM for the second, and 3 sets of 8 10RM for the third. This format was repeated for the second and third months as well. Recovery weeks for NLP and LP consisted of 3 sets 12RM on Mondays to 8RM on Wednesdays and 4RM on Fridays. Only the NLP group experienced significant bench press strength gains over the course of the 12 week training program with significant gains every 4 weeks. Increases in strength compared to NP and LP were significantly higher from baseline to week 8 and 12. NLP also resulted in significantly greater leg press strength compared to both NP and LP groups at every 4 week time point. NP saw no significant increases in leg press at any time point, but the LP group did experience significant strength increases from baseline to week 8 in the leg press, but no additional significant gains from week 8 to week 12 . Contrary to past findings, NP and LP groups were not significantly different from each other at any time point for either bench press or 
leg press in this study. Results of this study support the theory that non-linear, or undulating, periodization is superior for strength development, suggesting increased variability to training stimuli promotes greater adaptations.

A randomized control trial was conducted by Simäo et al. (2012). Thirty males who were habitually active, but had not resistance trained in the past 6 months, were randomly assigned to NLP, LP or a control group (CG: no resistance training). Muscle thickness via ultrasound of the right bicep and triceps as well as 1RM for bench press, lat pull-down, biceps curl, and triceps extension were measured on two non-consecutive days in a counterbalanced order before the start of the 12 week training program. Post testing was done in the same order 48 and 72 hours after the last session. LP training consisted of changes in volume and intensity every 4 weeks progressing from 2 sets of $12-15 \mathrm{RM}$ to 3 sets of 8-10RM and ending with 4 sets of 3-5RM. During weeks 1 through 6, NLP changed volume and intensity every two weeks using the same progression as LP. During weeks 7 through 12, each session's repetition schematic changed, rotating through the same progression from day to day. Subjects trained 2 days a week with 72 hours of rest between sessions and a warm up of 20 repetitions with only $50 \%$ of the load of their first set for each exercise. Statistical analyses showed no significant differences between groups at baseline or in volume lifted during training. After 12 weeks, NLP had significantly greater muscle thickness than CG for both triceps and biceps, but no group had significant increases from baseline. Both groups saw significant increases in strength for the lat pull down, bicep curl and triceps extension, but only NLP increased bench press strength. In addition, increases for NLP were significantly higher than LP. This study adds to the pool of research supporting the idea that the increased variability 
introduced in undulated periodization creates more frequent and erratic stimulation to the nervous system, facilitating greater strength adaptations than an LP design. While both NLP and LP result in increased strength, NLP has shown to be a more effective means of producing strength, with significantly greater gains than LP.

Undulated and Linear Equated. Research on the difference in effectiveness of UP vs. LP is still inconclusive when considering specific program variable manipulation. Miranda et al. (2011) is one of a few follow up studies comparing the two program types in a split routine format. Resistance trained subjects who had been actively training for the past 2 years were randomly assigned to either a DUP or LP strength program. No significant differences were found between groups in strength, mass, height, age, or experience between subjects at baseline. One-repetition maximum and 8RM of the leg press and bench press was tested pre-training on 4 separate occasions with 72 hours between each in a counterbalanced order. LP and DUP both consisted of two sessions: A, performed on Mondays and Thursdays, B, performed on Tuesdays and Fridays. Session A included bench press, chest fly, incline bench press, lateral raises, upright rows, shoulder press, triceps extension, barbell military press, and abdominal crunches. Session B was comprised of leg press, leg extension, leg curl, lat pull-down, seated row, reverse fly, biceps curl, preacher curl, and back extension. All exercises in both sessions, except the bench press and leg press, were performed in 3 sets of 6-8 RM. LP changed volume and intensity of leg press and bench press every 4 weeks progressing from 3 sets of 8-10, to 6-8RM, to 4-6 RM. DUP sessions rotated through these program schematics over the whole 12 weeks; for instance, session 1: 3x8-10RM, session 2:3x6-8RM, and session 3: 3x4-6RM. Before each training session, both programs included a warm up of 20 
repetitions of each exercise with $50 \%$ of the load to be used for the first set. Both LP and DUP experienced significant strength increases over the 12 weeks compared to baseline with no significant differences in volume between groups. While DUP showed greater strength values for bench press and leg press, they were not significantly different from LP. Miranda et al. (2011) propose statistical significance might not be the most appropriate means of determining which method is more effective when there is a small sample size. In this study, the standard deviation for mean strength gains were so large that effect size was thought to be a better measure of effectiveness, which revealed DUP to have a greater magnitude of 1RM and 8RM loads. Other studies of this kind however, had similar sample sizes and still produced significant findings. This study also utilized subjects who were habitually active and currently resistance trained which would decrease the possibility of seeing significant increases in strength compared to nontrained or detrained individuals.

\section{Conclusion}

Literature on Variability of Practice is extensive and the majority supports the theory that increased variability during practice is advantageous to overall learning levels. Based on the studies presented here, results demonstrate practicing 3 to 5 variations of a task in acquisition promotes learning of an enhanced motor response schema over constant practice of the same variations. This typical Variability of Practice paradigm utilized in

motor learning research however, has not been empirically examined in strength training programs. The idea that increased variability in program formats is more beneficial than classic linear periodization has been described in several articles and books. While frequency of varying load schematics every month or every few weeks has been 
examined, more recent research suggests more frequent alterations increase exposure to different stimuli, eliciting greater neural adaptations (Rhea et al., 2002; Monteiro et al., 2009; Siamo et al., 2012). Although research comparing undulating and linear periodization is not entirely conclusive, the preponderance of evidence suggests undulating periodization is more likely to provide strength increases compared to linear periodization. Examination of a more direct application of variable practice such as the present study, which varies volume and intensity within a single session, should provide more information on the extent to which variability of training improves strength gains. 


\section{CHAPTER III}

\section{Methods and Procedures}

\section{Purpose}

The primary purpose of this study was to examine whether variable practice facilitated enhanced neuromuscular adaptations when compared to traditional strength training. If variable practice facilitates greater neuromuscular adaptations by the mechanisms described, then the variable practice group will make greater strength gains than the traditional training by the end of the 8-week training program.

The secondary purpose was to investigate whether there was a difference in perception of exertion levels between the two strength training programs. If variable practice as described by Tsatsouline and John (2011) is a more efficient method of achieving adaptations than traditional weight lifting formats, then the variable practice treatment should produce lower RPE values than the traditional treatment.

\section{Dependent Measures}

To measure strength gains the ACSM 1RM test protocol was used. This method of assessing strength has been used in clinical, academic and research settings (ACSM, 2010; Miranda et al., 2011; Monteiro et al., 2009; Rhea et al., 2002; Simäo et.al, 2012). Subjects warmed up by completing at least 5 repetitions of the kettlebell press and leg press at $40 \%$ to $60 \%$ of a weight they thought they could complete multiple repetitions of. A 1-minute rest would follow with dynamic stretching of the used muscle groups. The next warm up set was comprised of at least 3 repetitions at $60 \%$ to $80 \%$ of their selfestimated 1RM, again followed by a 1-minute rest and stretching. The testing process 
began by estimating a possible 1RM weight and having the subjects attempt to complete 1 repetition. If the subject completed 1 repetition, after a rest period of 3 minutes a second trial was conducted with a weight increased by $1 \mathrm{~kg}$. This process was repeated until they could not complete a full repetition. A subject's 1RM was recorded as the weight lifted during the last successful trial.

Strength gains were calculated 3 different ways using subject's 1RM measures. Absolute strength gains at the first time point (T1) was the baseline 1RM subtracted from the 4 week 1RM, T2 was the 4 week 1RM subtracted from the 8 week 1RM, and T3 was the baseline 1RM subtracted from the 8 week 1RM, in kilograms. Percent strengths were calculated as the absolute gains at each time point divided by baseline 1RM values, multiplied by 100. In addition, subject's relative strength improvements were calculated as their 1RM at each time point divided by their body mass in kilograms.

Rating of perceived exertion (RPE) was also measured to assess if the level of intensity was perceived differently by subjects receiving different training. Borg's revised Category Ratio RPE (RPE) scale was used due to his application for exercise that induced localized exertion or fatigue to a particular part of the body (Day, McGuigan, Brice \& Foster, 2004). On the scale of 0 to 10,0 meaning rest and 10 being maximal exertion, an RPE of 1 to 4 is considered light exertion, 5 or 6 corresponding to moderate, and 7 to 9 as high exertion (Heyward, 2010). After each set was completed of each lift (kettlebell \& leg press), subjects were asked how many more repetitions they felt they could complete. RPEs were recorded as 10 if they replied with "zero" and 9 if they said 1, 8 if they said 2 etc., in order to streamline the protocol. For each lift, set RPEs were averaged to assess the subject's average RPE for the session. This was done to more appropriately compare 
differences between programs because subjects in the traditional treatment group only completed 3 sets in weeks one through six and 4 sets in weeks seven and eight, whereas subjects in the variable treatment group completed 5 sets each bout throughout the whole study. Session RPEs for each lift were then averaged to give subjects a single average lift RPE for all sessions.

\section{Design}

A total of 41 subjects, 23 women and 18 men, were assigned to either the control group or one of two treatment groups. A blocked-random method was employed to ensure similar ranges of relative strength within groups and equal average relative strength between groups. Relative volume of the variable and traditional training programs used as treatments were matched as well. Measurement of 1RM was conducted before assignment and subsequent training, as well as after 4 and 8 weeks. Progress 1RM testing sessions were scheduled 2 to 3 days after completing the 4th and 8th weeks of training depending on subject's availability. During training sessions, RPE was recorded after each set of the kettlebell press and leg press, as well as the session average RPE for both lifts. Absolute and percent strength gains, as well as relative strength improvements were calculated for the first 4 weeks, last 4 weeks, and the total 8 week time intervals. Strength gain data and RPE data were used for statistical analyses. Caffeine intake within 4 hours of training was recorded and used as a covariate in analyses as to not alter subject's training habits in relation to caffeine supplementation or dietary intake.

\section{Subjects and Recruitment}

Flyers were posted around the community in fitness centers, local college campuses, and local businesses asking for volunteers who weight lift regularly. Recruitment 
continued until 50 subjects, 24 males and 26 females, were screened and cleared based on the following inclusion/exclusion criteria: Each subject must have a) had weight lifting instruction with a qualified professional and at least 3 months of experience lifting weights habitually, b) been between the ages of 18 and 45, c) been classified as low risk unless cleared by their physician, d) not had any current, reoccurring, or chronic pain, limitations, or injuries and e) not been pregnant. Cardiac risk level was determined using ACSM's cardiac risk assessment (2010; Heyward, 2010). The eligibility screenings were conducted over the phone or in person based on convenience.

Subjects were also required to sign a "Subject's Agreement" form. Subjects in treatment groups were asked to refrain from any resistance training outside of the study and from discussing their training sessions with current or potential subjects. In contrast, control subjects were required to continue their current exercise regimen and not make any drastic changes over the course of the study duration. All subjects were required to refrain from consuming legal or illegal supplements and maintain their usual intake of caffeine for the duration of the study, as well as notify a researcher if they had become ill or injured. Only data of experimental participants who completed a minimum of 22 out of the 24 training sessions, and subjects who completed all testing sessions were included in data analysis.

Two males and 1 female were dropped due to incomplete baseline assessments, two more males due to an equipment malfunction before their final 1RM could be measured, and 1 for not completing the minimum number of required sessions. One female withdrew because she moved away half way through her sessions, and another had to be dropped for incomplete baseline assessments, and finally one for not completing all 
testing sessions. This resulted in the total of 41 subjects out of the 50 eligible subjects. Thus the final subject pool consisted of 18 males and 23 females.

\section{Procedures}

Once a subject's eligibility was established, the first familiarization session was scheduled. During the first familiarization session the researcher went over the health history questionnaire, informed consent, study procedures, and the "Subject Agreement" form. When the paperwork was complete, height, weight, and percent body fat $(\% \mathrm{BF})$ via the skinfold method were measured. Afterwards, the proper form for the kettlebell and leg press was taught. The second familiarization session was used to review the kettlebell and leg press techniques and allow test administrators to provide technique corrections and important cues before the 1RM tests. In this session the RPE scale was also introduced and used during the 1RM warm up protocol to help with understanding. When ready, the subject's kettlebell press and then leg press 1RMs were measured establishing baseline values for each subject.

Subjects were then assigned to either a control group or one of two treatment groups using a blocked random method, stratified for sex and relative strength to control for variability among groups. Next the subjects were contacted to schedule either their training sessions (treatment groups) or their next testing session (control group). The treatment groups completed 8 weeks of training (ACSM, 2010; Heyward, 2010; Rhea et al., 2002) consisting of 3 days a week with at least one recovery day in between each training session. Subjects were instructed to give their maximal effort for every repetition, and constrain both their concentric and eccentric actions to 3 seconds with 
only 1-2 seconds between repetitions. Three minutes of rest followed each set to ensure appropriate recovery (Heyward, 2010).

In weeks 1 through 3 , subjects undergoing the traditional treatment completed 3 sets of 8 repetitions at $70 \%$ of their 1RM for each exercise. Weeks 4 through 6 were composed of 3 sets of 6 repetitions at 77\% 1-RM. In weeks 7 and 8,3 sets of 4 repetitions, at $85 \% 1 \mathrm{RM}$ were performed.

Over the course of the eight weeks, the variable treatment was comprised of 5 sets of the kettlebell and leg press. A set of 4 repetitions at $77 \%$ of the subject's 1 RM, one of 5 repetitions at $70 \% 1 \mathrm{RM}$, another of 3 repetitions at $85 \% 1 \mathrm{RM}$, one of 6 repetitions at $65 \% 1 \mathrm{RM}$, and finally a set of 2 repetitions at $93 \% 1 \mathrm{RM}$ were performed every session. The order of these sets were changed every three weeks, altering where in the session the highest intensity set was in relation to the lower intensity sets.

Table 3.1. Training programs defined by specific set and repetition schematics

\begin{tabular}{lccc}
\hline Treatments & Weeks 1-3 & Weeks 4-6 & Weeks $7 \& 8$ \\
\hline \multicolumn{1}{c}{ Number of Sets \& Repetitions at Intensity, defined as a Percentage of Subject's 1RM } \\
\hline Traditional & $3 \times 8$ at $70 \%$ & $3 \times 6$ at $77 \%$ & $4 \times 4$ at $85 \%$ \\
Variable & $1 \times 4$ at $77 \%$ & $1 \times 3$ at $85 \%$ & $1 \times 5$ at $70 \%$ \\
& $1 \times 3$ at $85 \%$ & $1 \times 6$ at $65 \%$ & $1 \times 3$ at $85 \%$ \\
& $1 \times 5$ at $70 \%$ & $1 \times 2$ at $93 \%$ & $1 \times 4$ at $77 \%$ \\
$1 \times 6$ at $65 \%$ & $1 \times 4$ at $77 \%$ & $1 \times 2$ at $93 \%$ \\
$1 \times 2$ at $93 \%$ & $1 \times 5$ at $70 \%$ & $1 \times 6$ at $65 \%$ \\
\hline
\end{tabular}

Both programs included a warm up set consisting of 8 repetitions at $30 \% 1 R M$.

Additional exercises were performed during training sessions, but were not analyzed. Both training programs included the kettlebell Romanian deadlift and kettlebell bench rows, which were linearly periodized regardless of assigned treatment. After 4 weeks of training, the subjects retested their kettlebell and leg press 1RMs. Final assessments were 
conducted 2 to 3 days after the 8 week mark, which included height, weight, $\% \mathrm{BF}$, age, and 1RMs for all subjects.

\section{Statistical Analyses}

Repeated measure ANOVAs were conducted to compare the effect of treatment, time, and the treatment by time interaction on absolute and relative strength gains over the first 4 weeks, last 4 weeks, and after 8 weeks for both the kettlebell press and leg press. Changes in strength relative to subject's body mass at baseline, 4 weeks, and 8 weeks were analyzed in the same manner for both the kettlebell press and leg press. While treatments were stratified by sex and relative strength, these factors were included as covariates along with caffeine consumption. Follow up ANOVAs were run following significant F-values. Student t-tests and tukey follow-up comparisons were conducted following significant F-values found in ANOVAs. An alpha level of .05 was used to classify significant findings.

Separate analyses were conducted on data of the two treatments to evaluate RPE as a response variable as well as a covariate. The control group was not included as they did not participate in the training sessions. Repeated measure ANOVAs were conducted to compare the effect of treatment, time, and a treatment by time interaction on absolute and relative changes in $1 \mathrm{RM}$ as well as relative strength for both the kettlebell press and leg press with RPE included as another covariate. Separate ANOVAs for the kettlebell press and leg press were also run to compare the effects of treatment on average RPE using the same covariates. The effect of treatment over time on RPE was investigated similarly, with sex and session number as covariates. An alpha level of .05 was used to determine significance. 


\section{CHAPTER IV}

\section{Results}

\section{Restatement of Purpose and Hypotheses}

The primary purpose of this study was to examine whether variable practice facilitated enhanced neuromuscular adaptations when compared to traditional strength training. It was hypothesized that variable practice would facilitate greater neuromuscular adaptations resulting in greater strength gains when compared to a traditional training by the end of an 8-week strength training program.

The secondary purpose was to investigate whether there was a difference in perception of exertion levels between the two strength training methods. It was hypothesized that variable practice as described by Tsatsouline and John (2011) would elicit lower ratings of perceived exertion compared to the traditional strength training.

\section{Dependent Measures}

Subject's 1RMs for the kettlebell press and leg press were measured at baseline, after 4 weeks of training, and again after 8 weeks of training. The differences from each time point were calculated to assess absolute strength gains over particular time intervals. Strength gains made in the first 4 weeks (I1) was defined as the baseline 1RM subtracted from the week 4 1RM. Strength gains made in the last 4 weeks (I2) were calculated as the week 4 1RM subtracted from the week $81 \mathrm{RM}$, and strength gains made over the whole 8 week period (I3) were calculated as the baseline 1RM subtracted from the week $81 \mathrm{RM}$. Percent strength gains as a percentage of baseline 1RM values were then calculated.

Relative strength was also calculated, dividing $1 \mathrm{RM}$ values by each subject's mass. This 
measure is important because both male and female subjects were included in this study, which added more variability in mass across subjects. Thus relative strength allows for a comparison of absolute strength gains relative to mass.

Ratings of perceived exertion (RPE) were recorded following treatment to determine subject's perception of intensity during each set. Borg's revised Category Ratio RPE (RPE) 1 to 10 scale was used due to its application of localized exertion to a particular part of the body (Day, McGuigan, Brice \& Foster, 2004). After each set of each lift, subjects were asked how many more repetitions they felt they could complete. RPEs were recorded as 10 if they replied with "zero" and 9 if they said 1, 8 if they said 2 etc., in order to streamline the recording protocol. For each lift, all set RPEs were averaged to assess the subject's average RPE for the session. This was done to more appropriately compare differences between treatments because a different number of sets were completed in a session depending on subject's assigned treatment. Session RPEs of each subject, for each lift, were then averaged to give a single overall average RPE across all sessions.

\section{Subject Characteristics}

Statistical analyses were run using 41 subject's data, 15 of which were assigned to the variable treatment ( 7 males and 8 females), 14 were to the traditional treatment ( 6 males and 8 females), and 12 to the control group (5 males, 7 females). Analyses established there were no significant differences in height, weight, body fat percentage, or age of the subjects between groups at baseline or after 8 weeks. Weight and body fat percentage was analyzed and found no significant differences after 8 weeks compared to baseline. 
These results are displayed in table 4.1. This confirmed strength improvements were due to neurological adaptations rather than morphological which are related to increases in muscle mass.

Table 4.1. Subject characteristics

\begin{tabular}{lcccccc}
\hline Treatment & Age & Height $(\mathrm{cm})$ & $\begin{array}{c}\text { Baseline } \\
\text { Weight }(\mathrm{kg})\end{array}$ & $\begin{array}{c}\text { Post 8 week } \\
\text { Weight }(\mathrm{kg})\end{array}$ & $\begin{array}{c}\text { Baseline } \\
\text { Body Fat \% }\end{array}$ & $\begin{array}{c}\text { Post 8 week } \\
\text { Body Fat \% }\end{array}$ \\
\hline Variable & $25(5.8)$ & $170.9(9.4)$ & $70.5(9.9)$ & $70.7(9.7)$ & $21.0(6.7)$ & $21.1(7.5)$ \\
Traditional & $26(5.8)$ & $173.7(11.8)$ & $71.8(12.2)$ & $71.9(12.2)$ & $19.8(7.3)$ & $19.9(8.0)$ \\
Control & $27(6.9)$ & $171.6(10.3)$ & $73.0(16.4)$ & $73.1(15.5)$ & $20.1(7.3)$ & $21.1(7.7)$ \\
\hline
\end{tabular}

Values are means (SD)

\section{Time Effects on Strength}

Analyses of 1RM and relative strength data found significant increases in the kettlebell press and the leg press for all treatments. Kettlebell press 1RM increases for all treatments were significant at the 4-week mark compared to baseline and the 8-week mark compared to 4 weeks and baseline. The treatment groups, both variable and traditional, leg press 1RM data showed significant increases over the first 4 weeks, the last 4 weeks, and over the whole 8 weeks. The control group did not experience significant increases after the first 4 weeks, but did over the last 4 weeks and all 8 weeks. Relative strength increased significantly in the kettlebell press and the leg press for both treatment groups after the first four weeks, and again after the last 4 weeks. The control group only made significant relative strength increases after all 8 weeks in the kettlebell press and after all 8 weeks compared to week 4 and baseline in the leg press. Data regarding 1RM and relative strength increases can be viewed in table 4.2. 
Absolute strength analyses found the kettlebell press gains made by all groups over the last 4 weeks (I2) were significantly greater than the gains made over the first 4 weeks (I1). Absolute strength gains made over the entire 8 weeks (I3) were greater than the gains made in both the first and last 4 weeks. Leg press absolute strength gains made by the traditional treatment were significantly greater over I 3 compared to I 2 and I1 whilst the control group's gains were significantly greater over I2 and I3 compared to I1. Absolute strength gains made by the variable treatment were significantly greater over I2 compared to I1, and over I3 compared to I2 and I1, making it the only treatment that made significantly greater strength gains over every time interval. Percent strength gains were significantly greater for the kettlebell press over I3 compared to I1 for all treatments. Both treatment groups also made significantly greater percent gains over I3 compared to I2. Similarly, percent strength gains in the leg press were significantly greater over I3 compared to I1 for all groups. However, significantly greater gains were made by both treatment groups over I3 compared to I2, and over I 2 compared to I1. So while all groups made various increases in strength over time, the variable and traditional groups made substantially greater strength gains than the control group from each time point to the next. Results are depicted in Table 4.2 and 4.3. 
Table 4.2. Mean 1RM (kg) measures and relative strength $(1 \mathrm{RM} / \mathrm{BW})$ over time

\begin{tabular}{|c|c|c|c|}
\hline 1RM Assessments & Baseline & Week 4 & Week 8 \\
\hline \multicolumn{4}{|c|}{ Kettlebell Press $[\mathrm{kg}(\mathrm{SD})]$} \\
\hline Variable & $19.9(5.3)$ & $21.4(6.0) \dagger$ & $22.8(6.5) \dagger$ \\
\hline Traditional & $21.0(7.7)$ & $22.3(8.1) \dagger$ & $23.7(8.4) \dagger$ \\
\hline Control & $20.7(7.6)$ & $21.5(8.1) \dagger$ & $22.2(8.4) \dagger$ \\
\hline \multicolumn{4}{|l|}{ Leg Press $[\mathrm{kg}(\mathrm{SD})]$} \\
\hline Variable & $180.9(44.9)$ & $229.5(56.5) \dagger$ & $257.0(59.3)+\vdots$ \\
\hline Traditional & $202.3(85.7)$ & $242.9(90.9) \dagger$ & $270.6(102.1) \dagger$ \\
\hline Control & $218.7(100.1)$ & $223.0(87.5)$ & $248.1(50.3) \dagger$ \\
\hline Relative Strength & Baseline & Week 4 & Week 8 \\
\hline \multicolumn{4}{|c|}{ Kettlebell Press [kg(SD)] } \\
\hline Variable & $0.28(.06)$ & $0.30(.07) \dagger$ & $0.32(.08) *+$ \\
\hline Traditional & $0.29(.07)$ & $0.30(.08) \dagger$ & $0.33(.09)^{*}+\hbar$ \\
\hline Control & $0.28(.07)$ & $0.29(.07)$ & $0.30(.08) \dagger$ \\
\hline \multicolumn{4}{|l|}{ Leg Press $[\mathrm{kg}(\mathrm{SD})]$} \\
\hline Variable & $2.6(.58)$ & $3.3(.70) * \dagger$ & $3.6(.83)^{*+t}$ \\
\hline Traditional & $2.8(.91)$ & $3.3(.88)^{*} \dagger$ & $3.7(1.0)^{*}+\hbar$ \\
\hline Control & $2.9(1.0)$ & $3.0(.79)$ & $3.3(.99)+t$ \\
\hline
\end{tabular}

\section{Treatment Effects on Strength}

Baseline 1RM and relative strength values for the kettlebell and leg press were not significantly different for any treatment. After training, there were no significant differences in kettlebell press or leg press 1RM values for any treatment after 4 or 8 weeks. Relative strength in the kettlebell press was not significantly different between treatments after 4 weeks; however both variable and traditional training resulted in significantly greater increases compared to the control group after 8 weeks. Variable and traditional training also resulted in significantly greater leg press relative strength after 4 and 8 weeks compared to the control group. Results are depicted in table 4.2.

Absolute strength gains in the kettlebell press were not significantly different between treatments over any time interval. Both the variable and traditional treatments made 
significantly greater leg press absolute strength gains than the control group after 4 weeks. After 8 weeks, the variable training showed significantly greater absolute strength gains in the leg press than the control group, while the traditional training did not (figure 4.2). Although gains made by the variable treatment were not significantly different from that of the traditional treatment, variable training did result in a greater mean strength increase. Furthermore, the variable treatment had greater mean strength gains over every time interval in comparison to the traditional treatment, except for absolute leg press and percent kettlebell press over the last 4 weeks (Depicted in table 4.3.). Both the variable and traditional training resulted in significantly greater percent strength gains in the leg press after 4 weeks (figure 4.4), and the kettlebell press after 8 weeks compared to the control group (figure 4.3). Strength gains made by the variable and traditional treatments were not however, significantly different. Data for these results are in table 4.3. 
Table 4.3. Mean absolute and percent strength gains (change in 1RM) across time

\begin{tabular}{cccc}
\hline Absolute Gains & I1 & I2 & I3 \\
\hline Kettlebell Press [kg(SD)] & & & \\
Variable & $1.5(1.4)$ & $1.4(1.1)$ & $2.9(2.4) \dagger$ \\
Traditional & $1.3(1.1)$ & $1.3(1.0)$ & $2.6(1.6) \dagger$ \\
Control & $0.78(1.2)$ & $0.64(.93)$ & $1.4(1.6) \dagger$ \\
Leg Press [kg(SD)] & & & \\
Variable & $48.6(19.4)^{*}$ & $27.6(15.7) \dagger$ & $76.1(29.0)^{*} \dagger \dagger$ \\
Traditional & $40.6(30.1)^{*}$ & $27.7(22.2)$ & $68.3(49.4) \dagger$ \\
Control & $4.3(25.2)$ & $25.1(24.9) \dagger$ & $29.4(35.4) \dagger$ \\
\hline Percent Gains & $\mathrm{I} 1$ & $\mathrm{I})$ & $\mathrm{I}$ \\
\hline Kettlebell Press [\%(SD)] & & & \\
Variable & $7.4(6.1)$ & $6.8(6.0)$ & $14.2(10.7)^{*} \dagger \dagger$ \\
Traditional & $6.4(4.6)$ & $7.3(6.5)$ & $13.6(8.6)^{*} \dagger \dagger$ \\
Control & $3.4(6.1)$ & $3.1(4.2)$ & $6.5(7.3) \dagger$ \\
Leg Press [\%(SD)] & & & \\
Variable & $27.2(10.5)^{*}$ & $16.5(11.7) \dagger$ & $43.7(19.4) \dagger$ \\
Traditional & $24.2(19.3)^{*}$ & $15.6(15.0) \dagger$ & $39.8(32.7) \dagger$ \\
Control & $6.2(15.3)$ & $11.3(7.8)$ & $17.5(18.5) \dagger$ \\
\hline
\end{tabular}

I1: First 4 weeks; I2: Last 4 weeks; 13: All 8 weeks

*Significantly greater compared to control; + Significantly greater compared to II;

\# Significantly greater compared to I2; significance is $p<.05$

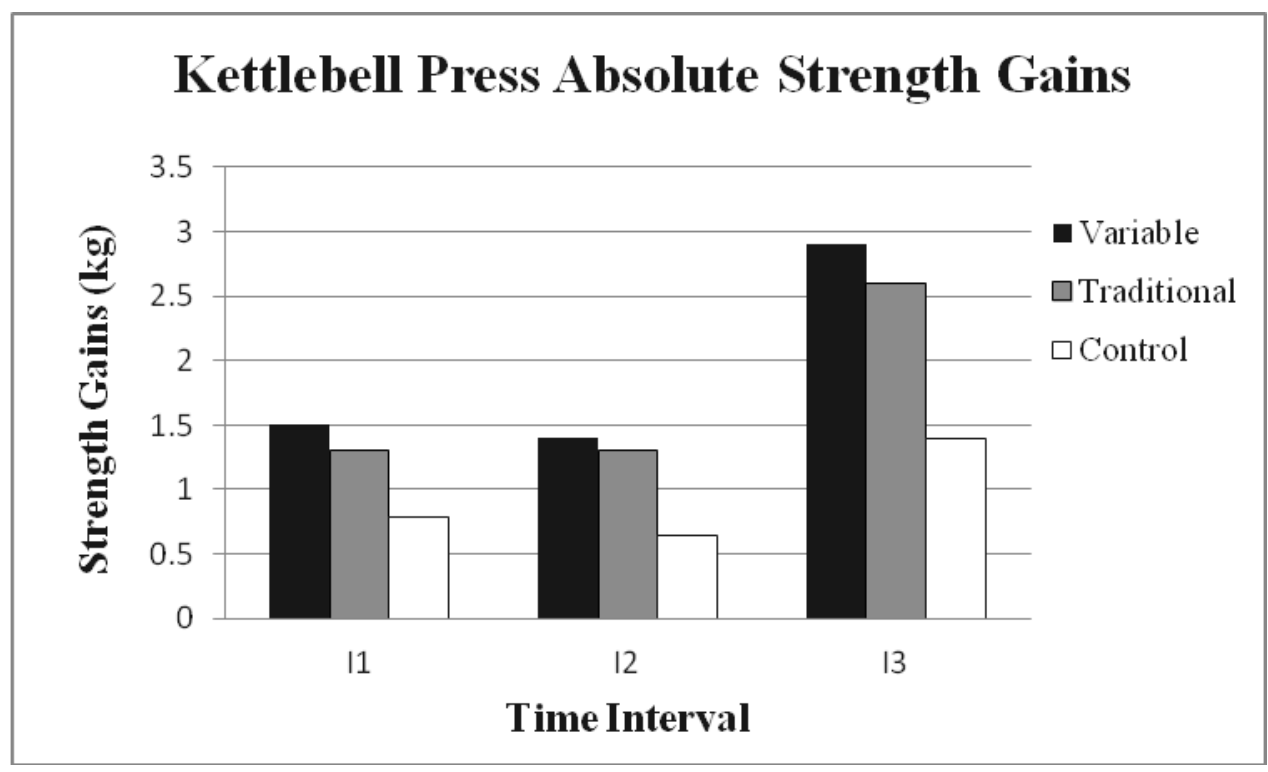

Figure 4.1. Absolute strength gains made in the kettlebell press by all groups.

Values are changes in $1 \mathrm{RMs}(\mathrm{kg})$ over the first 4 weeks of training (I1), the second 4 weeks of training (I2) and the entire 8 weeks (I3). 


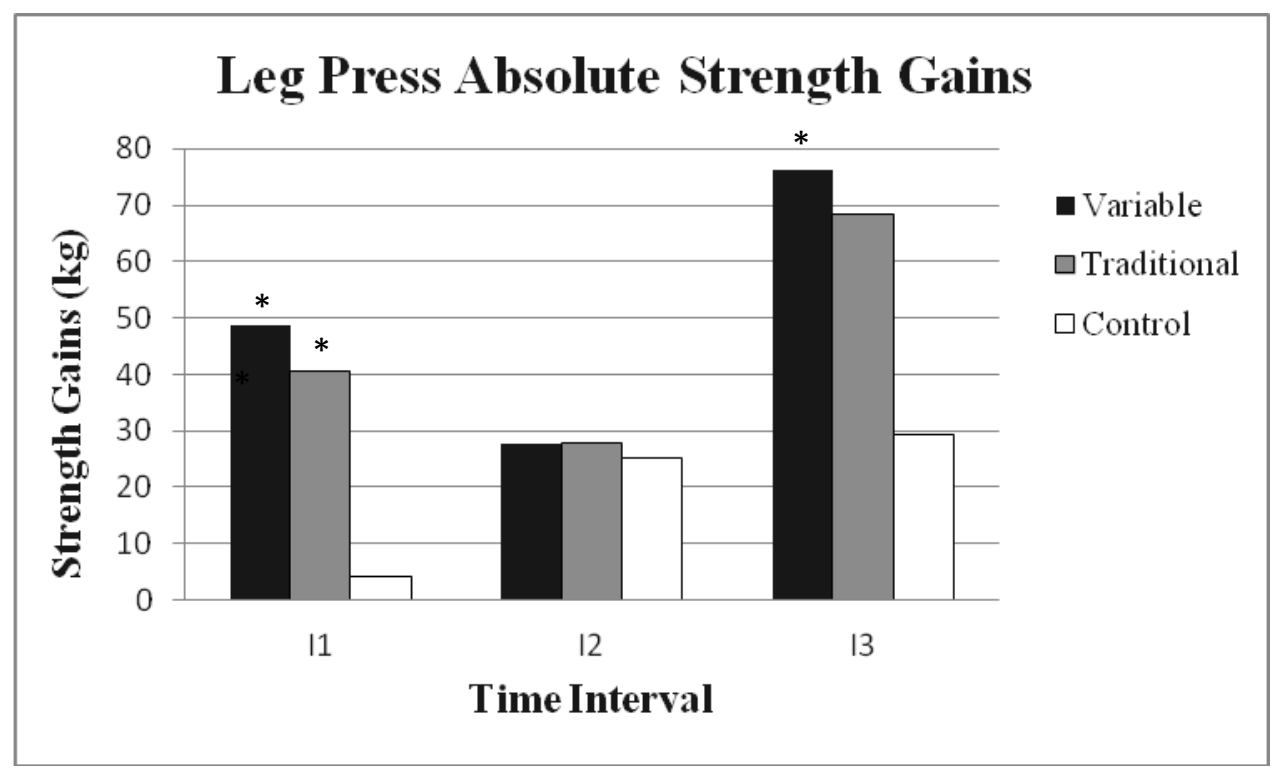

Figure 4.2. Absolute strength gains made in the leg press by all groups. Values are changes in 1RMs (kg) over the first 4 weeks of training (I1), the second 4 weeks of training (I2) and the entire 8 weeks (I3). *Significantly $(\mathrm{p}<.05)$ greater gains compared to the control group.

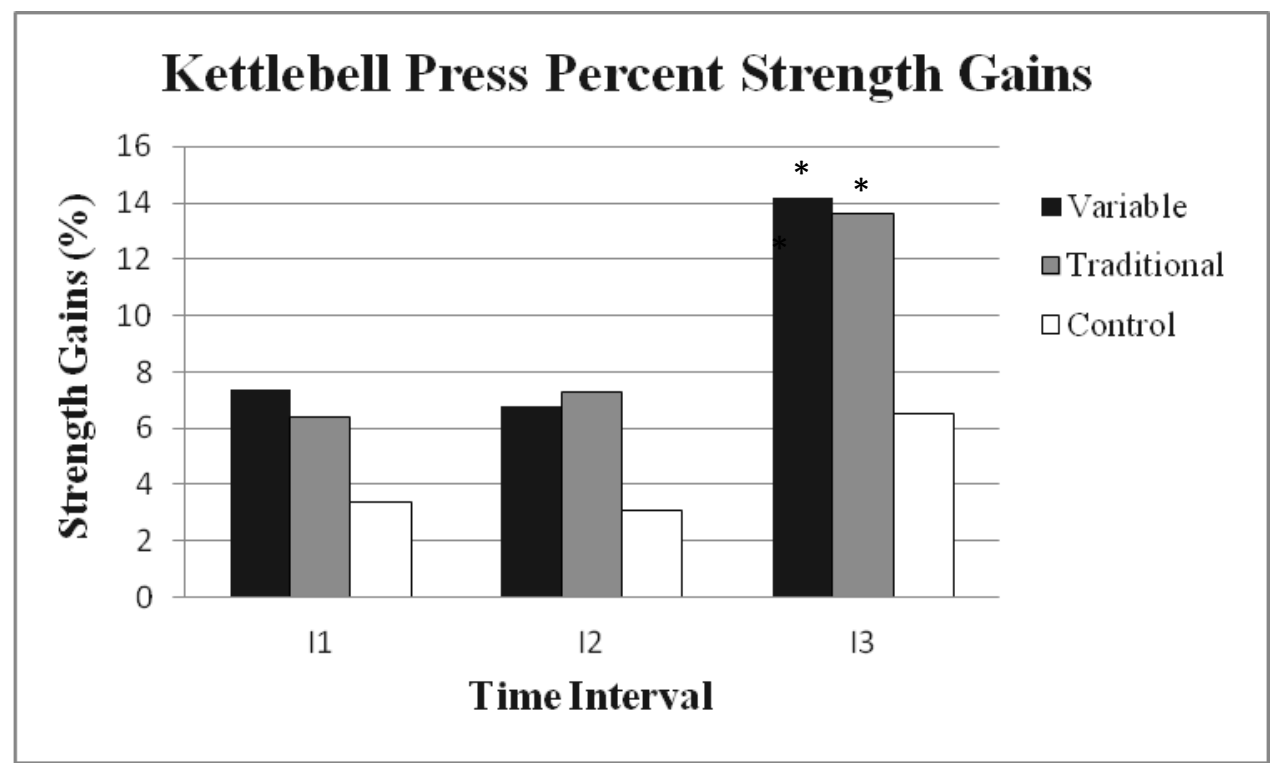

Figure 4.3. Percent strength gains made in the kettlebell press by all groups.

Values are changes in $1 \mathrm{RMs}(\mathrm{kg})$ divided by baseline $1 \mathrm{RM}$ values and multiplied by 100 , over the first 4 weeks of training (I1), the second 4 weeks of training (I2) and the entire 8 weeks (I3). *Significantly $(\mathrm{p}<.05)$ greater gains compared to the control group. 


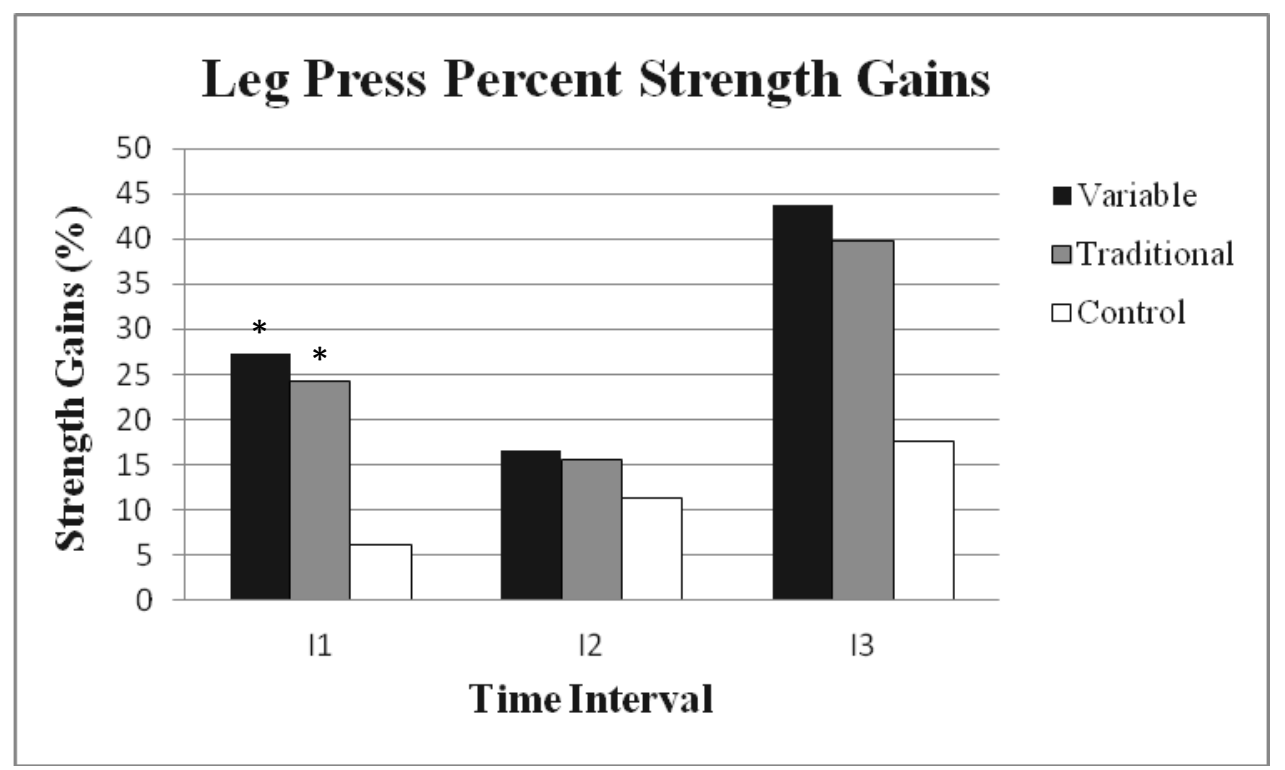

Figure 4.4. Percent strength gains made in the leg press by all groups. Values are changes in $1 \mathrm{RMs}(\mathrm{kg})$ divided by baseline $1 \mathrm{RM}$ values and multiplied by 100 , over the first 4 weeks of training (I1), the second 4 weeks of training (I2) and the entire 8 weeks (I3). *Significantly $(\mathrm{p}<.05)$ greater gains compared to the control group.

Table 4.4. Repeated Measure ANOVA Alpha Levels

\begin{tabular}{lcccccc}
\hline \multirow{2}{*}{ Main Effect } & \multicolumn{3}{c}{ Kettlebell Press Strength Gains } & \multicolumn{3}{c}{ Leg Press Strength Gains } \\
\cline { 2 - 7 } & Absolute & Percent & Relative & Absolute & Percent & Relative \\
\hline Treatment & 0.0571 & $0.0319^{*}$ & $0.0227^{*}$ & $0.0054^{*}$ & $0.0136^{*}$ & $0.0020^{*}$ \\
Time & $0.0183^{*}$ & $0.0007^{*}$ & 0.6362 & $0.0323^{*}$ & $0.0023^{*}$ & $<.0001^{*}$ \\
Treatment*Time & 0.0740 & 0.0672 & $0.0231^{*}$ & 0.9697 & 0.8103 & $0.0004^{*}$ \\
\hline KB: Kettlebell Press, LP: Leg Press; significance is $p<.05$ &
\end{tabular}

\section{Ratings of Perceived Exertion Effects and Responses}

Data analyses revealed that subjects with lower reported ratings of perceived exertion (RPE) made significantly greater absolute strength gains over the last 4 weeks and over the entire 8 weeks for both the kettlebell and leg press regardless of assigned treatment. Similarly, subjects who reported lower kettlebell press RPE values made significantly greater percent strength gains over the 8 weeks than those who reported higher RPEs. In 
the leg press, lower reported RPE values resulted in greater percent strength gains over every time interval (Significant p-values can be viewed in table 4.5.). A significant sex by RPE interaction revealed males and females had significantly different kettlebell strength gains in response to reported RPEs. Absolute and percent strength gains made over the first 4 weeks (I1) were significantly greater for male subjects who reported lower RPEs than male subjects who reported higher RPEs. Kettlebell press relative strength after 4 weeks was also significantly higher for male subjects who reported lower RPEs compared to male subjects who reported higher RPEs. Although this relationship was present for female data, the effect of reported RPEs was not significant on resultant strength gains. This interaction suggests that females may achieve similar strength gains regardless of their perceived level of exertion as compared to males. Therefore, males may benefit more than females from strength training programs perceived as less difficult.

Table 4.5. Alpha levels of RPE effects on strength gains for treatment groups

\begin{tabular}{|c|c|c|c|}
\hline Strength Increases & $\mathrm{I} 1(\mathrm{p})$ & I2(p) & $\mathrm{I} 3(\mathrm{p})$ \\
\hline \multicolumn{4}{|l|}{ Absolute Gains } \\
\hline Kettlebell Press RPE & 0.0651 & $0.0332 *$ & $0.0127 *$ \\
\hline Leg Press RPE & 0.0916 & $0.0170^{*}$ & $0.0271^{*}$ \\
\hline \multicolumn{4}{|l|}{ Percent Gains } \\
\hline Kettlebell Press RPE & 0.0958 & 0.0622 & $0.0256^{*}$ \\
\hline Leg Press RPE & $0.0815^{*}$ & $0.0122 *$ & $0.0140^{*}$ \\
\hline Relative Strength & \multicolumn{2}{|c|}{ Week 4(p) } & Week 8(p) \\
\hline Kettlebell Press RPE & \multicolumn{2}{|c|}{0.0818} & $0.0141^{*}$ \\
\hline Leg Press RPE & \multicolumn{2}{|c|}{$0.0204 *$} & $0.0048^{*}$ \\
\hline
\end{tabular}

*Significance is $p<.05$

Analyses examining RPE as a response variable found significant treatment effects as variable training resulted in significantly lower reported RPEs, for both the kettlebell and leg press compared to traditional training. These results are depicted in figures 4.5 and 
4.6 with data displayed in table 4.6. There was also a significant interaction $(\mathrm{p}=0.0434)$ of treatment by sex, revealing traditional female subjects reported significantly higher RPE values than variable females. Additionally, when RPE was analyzed over time, reported RPEs for the leg press were found to have significantly $(p=0.0146)$ increased throughout the study while reported RPE for the kettlebell press did not.

Table 4.6. Training session mean RPEs

\begin{tabular}{lc}
\hline Treatment & Session RPE \\
\hline Kettlebell Press [RPE(SD)] & \\
Variable & $7.83(0.27)^{*}$ \\
Traditional & $8.42(0.24)$ \\
Leg Press [RPE(SD)] & \\
Variable & $7.30(0.29)^{*}$ \\
Traditional & $7.66(0.45)$ \\
\hline *Significantly $(p<.05)$ & lower than traditional
\end{tabular}

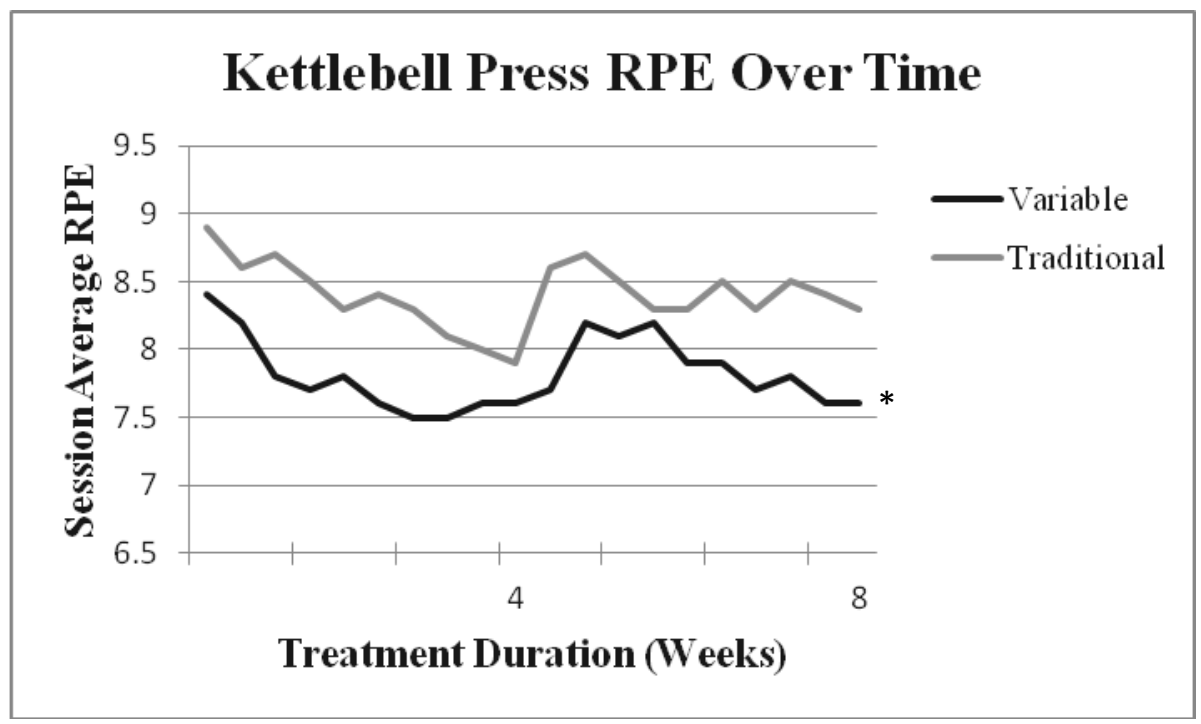

Figure 4.5. Mean reported ratings of perceived exertion (RPE) for the kettlebell press over both 8 week treatments. Values are based on a scale of 1 to 10,10 being maximum exertion. *Significantly $(\mathrm{p}<.05)$ lower reported RPEs. 


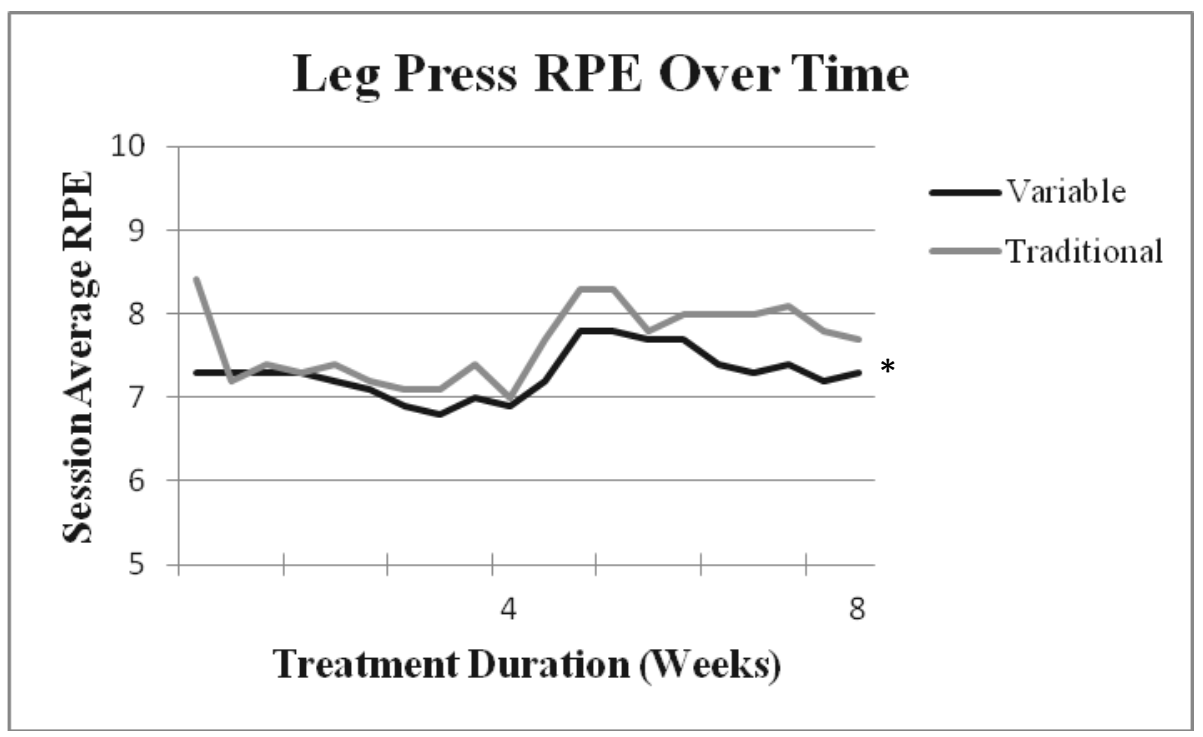

Figure 4.6. Mean reported ratings of perceived exertion (RPE) for the leg press over both 8 week treatments. Values are based on a scale of 1 to 10,10 being maximum exertion. ${ }^{*}$ Significantly $(\mathrm{p}<.05)$ lower reported RPEs.

\section{Sex Differences}

Over the course of the study, both males and females significantly increased their relative strength for the kettlebell press and leg press every 4 weeks. Males were significantly stronger than females in the kettlebell press at baseline and after 8 weeks, whereas only at baseline in the leg press. Both males and females made significantly greater absolute and percent strength gains in the kettlebell press over I 3 compared to I 2 and I1, with males having made significantly greater gains than females over I3 and I2. Males and females both achieved significant absolute and percent strength gains in the leg press over I 3 compared to I 2 and I1, but only the females had significant increases over I2 compared to I1. Males were found to have made significantly greater absolute leg press strength gains than females over I2 and I3, but not percent gains. Interestingly, although not significant, females made greater percent strength gains in the leg press than 
males over I1, I2, and I3. There was also a significant interaction of treatment by sex showing male control subjects made greater absolute strength gains in the leg press over I2 than female control subjects. Sex differences data can be viewed in table 4.7.

Table 4.7. Strength gains and relative strength increases by sex

\begin{tabular}{|c|c|c|c|}
\hline Relative Strength & Baseline & Week 4 & Week 8 \\
\hline \multicolumn{4}{|l|}{ Kettlebell Press } \\
\hline Males & $0.34(.05)^{*}$ & $0.36(.06) \dagger$ & $0.39(.06)^{*+\dagger}$ \\
\hline Females & $0.24(.04)$ & $0.25(.04) \dagger$ & $0.26(.04) \dagger t$ \\
\hline \multicolumn{4}{|l|}{ Leg Press } \\
\hline Males & $3.36(.69)^{*}$ & $3.80(.59) \dagger$ & $4.26(.67) \dagger+$ \\
\hline Females & $2.25(.57)$ & $2.72(.56) \dagger$ & $3.01(.73) \dagger+$ \\
\hline Strength Gains & I1 & $\mathrm{I} 2$ & I3 \\
\hline \multicolumn{4}{|l|}{ Kettlebell Press } \\
\hline \multicolumn{4}{|c|}{ Absolute Gains (kg) } \\
\hline Males & $1.78(1.5)$ & $1.69(1.1)^{*}$ & $3.48(2.1) * \dagger+$ \\
\hline Females & $0.78(.88)$ & $0.70(.81)$ & $1.49(1.3) \dagger+$ \\
\hline \multicolumn{4}{|c|}{ Percent Gains (\%) } \\
\hline Males & $6.97(6.2)$ & $6.76(5.4)^{*}$ & $13.73(10.1)^{* \dagger \dagger}$ \\
\hline Females & $4.98(5.3)$ & $5.21(6.3)$ & $10.18(8.9) \dagger \dagger$ \\
\hline \multicolumn{4}{|l|}{ Leg Press } \\
\hline \multicolumn{4}{|c|}{ Absolute Gains (kg) } \\
\hline Males & $34.91(39.3)$ & $34.26(19.9)^{*}$ & $69.17(46.4) * \dagger t$ \\
\hline Females & $31.32(23.4)$ & $21.14(19.4) \dagger$ & $52.46(39.4) \dagger+$ \\
\hline \multicolumn{4}{|c|}{ Percent Gains (\%) } \\
\hline Males & $15.27(16.5)$ & $13.02(6.9)$ & $28.30(20.0) \dagger \dagger$ \\
\hline Females & $23.74(17.7)$ & $15.98(14.7) \dagger$ & $39.72(30.1)+t$ \\
\hline
\end{tabular}

* Significantly greater than females; + Significantly greater compared to I1;

$¥$ Significantly greater compared to I2; significance is $p<.05$

\section{Caffeine Effects}

A significant difference due to caffeine consumption was only found at 8 weeks.

Results revealed those who did not consume caffeine had significantly ( $\mathrm{p}$ value $<0.0133$ ) greater relative strength in the kettlebell press than the caffeine consumers. Additionally, effects of caffeine on RPE responses demonstrated that the caffeine consumers reported 
significantly higher ratings of perceived exertion for the kettlebell press than the non caffeine consumers seen in table 4.8 .

Table 4.8. Alpha levels of caffeine use within 4 hours of training sessions

\begin{tabular}{cc}
\hline Lift & Effect on Average RPE (p) \\
\hline Kettlebell Press & $0.0328^{*}$ \\
Leg Press & 0.0901 \\
\hline * significance is $p<.05$
\end{tabular}




\section{CHAPTER V}

\section{Discussion and Conclusions}

The present study examined a direct application of variable practice to strength acquisition. In the motor learning literature, cognitive processing theorists suggest that practicing multiple variations of a task concurrently facilitates the learning of those tasks. This occurs by creating a more challenging cognitive processing environment during practice. The mechanism suggested by Schmidt was that variable practice enhances the development of the motor response schemas which allow for parameter refinement. Better parameter control contributes to greater task performance during future endeavors (Schmidt, 1975; 1988). Recently strength specialists have recommended increased variability within strength training programs (John \& Tsatsouline, 2011; Rhea et al., 2002). However, a direct application of the Variability of Practice paradigm in a strength training setting has not been examined empirically. While frequency of changes in volume and intensity have been examined in studies using undulating periodization (Monteiro et al., 2009; Siamo et al., 2012) and daily undulating periodization (Rhea et.al, 2002; Miranda et al., 2011), evidence suggests that the more frequent the variation, the more likely neurological strength increases will ensue.

In the present study, program components such as number of sets, number of repetitions per set, and intensity of each set were arranged differently for the treatment groups. To apply the Variability of Practice Hypothesis to strength training, treatments were designed to represent variable practice and constant practice, while matching total relative volume of both treatments. For the variable treatment, program components were arranged so every set was different. Subjects completed 5 total sets, each consisting of a 
different number of repetitions at different percentages of their 1RM. For the traditional treatment, these same program components remained constant, i.e., 3 sets of 8 repetitions at $70 \% 1 \mathrm{RM}$. Over the course of the program, these schemes were rearranged every 3 weeks to equate total relative volume between treatments. The present study introduces variability within every training session. This is unique compared to the programs used in strength literature in which programming components are altered by day or by week.

Empirical support for Variability of Practice within a strength training setting is limited. Nevertheless, support is derived from studies that utilized an undulating or daily undulating periodization program. Strength programs used as treatments in previous studies varied volume and intensity similarly to the present study, the difference being the frequency of variation. Undulating periodization changes the training components described previously every 1 to 2 weeks. For example the first two weeks may consist of 3 sets of 10 repetitions at $65 \% 1 \mathrm{RM}$, and then change for weeks three and four to 3 sets of 8 repetitions at $70 \% 1 \mathrm{RM}$. Generally each time the training scheme changes, volume would decrease while intensity increased for the duration of the program. Volume is calculated as the number of sets multiplied by the number of repetitions completed, multiplied by the weight lifted. Intensity is denoted by the percentage of the individual's $1 \mathrm{RM}$ at which the set is performed. In daily undulating periodization, training components are changed each day within the week. So day one of each week may be composed of 3 sets of 5 repetitions at $75 \% 1 \mathrm{RM}$, then 4 sets of 3 repetitions at $80 \% 1 \mathrm{RM}$ on day 2, whereas day 3 may be 4 sets of 3 repetitions at $85 \% 1 \mathrm{RM}$. Each day would follow the standard trend within a periodized program of decreasing volume and increasing intensity. This scheme would repeat each week for the duration of the 
program. Thereby, treatments used presently could be identified as session undulating periodization when compared to previous literature examining Variability of Practice in strength training settings.

\section{Strength Gains}

Results of this study demonstrate that for all treatments, significant increases in overall strength were achieved. When comparing the control group to treatment groups, both the variable and traditional treatments achieved significantly greater relative strength in the kettlebell and leg press than the control group. Statistical analyses did not support the primary hypothesis that the variable treatment would facilitate greater strength gains than the traditional treatment after 8 weeks. However, the variable treatment did make significantly greater leg press absolute strength gains than the control group. Although

gains made by the variable treatment were not significantly different from the traditional treatment's, variable training did result in a greater mean strength increase. Furthermore, the variable treatment had greater mean strength gains over almost every time interval in comparison to the traditional treatment, demonstrating a trend in favor of variable training benefits. The proposed mechanism supporting this statement involves neurological estimation of force production. The variable treatment forces the neurological system to approximate forces necessary to successfully lift several different weights each session. In addition, the order of weight lifted is waved so sets of lower intensity are followed by sets of higher intensity. Conversely, the traditional treatment only requires subject's neurological system to assess needed force requirements for a single weight each session. Thereby, exposure to multiple force parameters for varying 
weights result in neurological adaptations to handle a range of weights rather than a single weight (Enoka, 2006; Gabriel et al., 2006; John \& Tsatsouline, 2011).

Another possible explanation for the variable treatment's greater mean increases in strength is that the variable group performed sets of higher intensity reaching up to $93 \% 1 \mathrm{RM}$ compared to the traditional treatment's $85 \% 1 \mathrm{RM}$. The idea that higher intensity sets elicit greater neuromuscular development is primarily anecdotal rather than empirical (Aarskog, Wisnes, Wilhelmsen, Skogen, \& Bjordal, 2011). However, theories such as the size principle do state that for maximal motor recruitment to occur, greater loads are required (Cormie, et al., 2011). Thereby adaptations such as greater intra and intermuscular synchronization occur maximizing motor unit recruitment and firing capacities (Duchateau, Semmler, \& Enoka, 2006; Gabriel et al., 2006). Compared to the traditional treatment, the dispersion of loading over 5 sets decreases the amount of time muscles are under tension. As both treatments were provided the same amount of rest between sets, the variable treatment offers a greater recovery to stress ratio, thus allowing greater potential for maximum effort each set. In theory, variable treatment would develop greater ability and accuracy to evoke the necessary force to lift a novel, or perhaps heavier, weight. While these notions are not unequivocally supported by the findings of the current study, they advocate for the effectiveness of Variability of Practice in a strength training setting.

\section{Ratings of Perceived Exertion}

Rating of perceived exertion (RPE) is a subjective measure reported by subjects regarding perceived effort during a set. The treatment's effect on RPE was found to be 
significant with the variable treatment reporting significantly lower RPE values in both the kettlebell press and the leg press than the traditional treatment. An interaction of treatment by sex reached significance as the variable female subjects reported significantly lower RPEs than the traditional female subjects. Interestingly, while relative volume between treatments were matched, the variable treatment perceived their sessions as less difficult to complete. These findings support the secondary hypothesis of this study, and John and Tsatsouline's (2011) proposed theory that increased variability is less stressful on the neuromuscular system than traditional training formats. The perception of exertion reported by the variable treatment subjects was significantly lower than the traditional subjects, while making similar overall strength gains. The distribution of sets and their relative volumes in the variable treatment could account for lower ratings of perceived exertion. Variable subjects completed a range of repetitions from 2 to 6 over 5 sets within every session. On the other hand, the traditional treatment performed the same number of repetitions each session; 8 the first three weeks over 3 sets, 6 the second three weeks, and 4 repetitions over 4 sets the last two weeks. Therefore, the amount of time muscles are under tension during the variable treatment is shorter on average compared to during traditional treatment. Volume in a single session is also dispersed over more sets in variable treatment, providing more total rest time than the traditional treatment as both treatments were given 3 minutes of rest between sets. Another attribute of the variable treatment is waving of set intensities in which sets of lower intensity preceded sets of higher intensity. Potentially, performing sets perceived as easier may provide the confidence in succeeding sets of higher intensity due to a greater sense of recovery prior. Together these proposed mechanisms all related to a positive increase in the recovery to 
stress ratio of variable training compared to traditional schemes. As seen here, there are many components to program schematics that could be responsible for the present RPE results; however research on each of these component's effect on RPE is lacking. Future research is needed to pinpoint the optimal program design for reducing RPE while including enough neuromuscular stimulation to facilitate strength adaptations.

At several time points, lower reported RPEs were found to be associated with greater strength gains. For both the kettlebell press and leg press, analyses found that greater absolute and percent strength gains, as well as relative strength increases, occurred when lower RPE values were reported. These, results suggest greater strength gains can be achieved by training methods perceived as less strenuous, implying that variable training may be an "easier" method to augment strength gains as compared to traditional training. A possible explanation for this is that individuals who were less exhausted were more able to perform sets with greater effort and integrity. For example, subjects were instructed to constrain concentric and eccentric movements to 3 seconds. This is to ensure slow progressive tension build up believed to increase strength by recruiting the most motor units as possible (Cormie, et al., 2011; Crewther, Keogh, Cronin, \& Cook, 2006). Those who were more tired or perceived each set as very difficult may have had a harder time keeping a slow consistent pace, failing to recruit as many motor units are those who had more energy. Care was taken to keep subjects performance consistent between and within groups, however, time it took subjects to complete the concentric and eccentric movements of each lift were not recorded; so this variable cannot be analyzed to confirm or refute this prediction. 
Significant sex by RPE interactions revealed that males and females had significantly different kettlebell strength gain results in response to reported RPEs. Analysis of both absolute and percent strength gains made over the first 4 weeks found males who reported lower RPEs achieved greater gains than male subjects who reported higher RPEs. Those male subjects who reported lower RPEs also had higher kettlebell relative strength at 4 weeks compared to those who reported higher RPEs. Female subjects on the other hand did not have as drastic of a relationship between strength gains and RPE. There were no significant sex by RPE interactions revealing differences in reported RPE between the sexes, therefore, this interaction cannot merely be attributed to a difference in perception of effort by either sex. This suggests that females may achieve increases in strength regardless of the perceived difficulty of the training, while males may benefit more from a strength program designed to reduce perceived exertion.

\section{Sex Differences}

Males and females both increased strength similarly over the different time intervals, contributing to the collective strength gain data. Male subjects, however, made greater absolute strength gains in both the kettlebell and leg press over the last 4 weeks of training as well as over the total 8 weeks. Kettlebell percent strength gains and relative strength were also significantly higher for males; leg press percent strength gains on the other hand were not significantly different over any time interval. These results are supported by research specific to sex differences in response to resistance training (Kell, 2011). R. T. Kell explains that upper body muscle groups differ in development from lower body musculature due to the anti-gravity function of hip and knee flexors and extensors as well as their constant use during daily living activities. It is also common for 
females to make greater percent increases in strength than males simply due to their lower baseline relative strength, as this increases the capacity to make improvements ( $\mathrm{p}$. 148). A significant interaction revealed control males made greater absolute strength gains in the leg press compared to control females. Differences in strength gains is most likely due the type of exercise control subjects participated in, as males reported higher incidence of weight training and females participated in more cardiovascular activities.

\section{Caffeine}

Caffeine consumption data was collected simply as a control measure. The effect of caffeine was not significant over the 8 weeks except at one time point. Subjects who consumed caffeine within 4 hours of training had significantly lower relative strength in the kettlebell press after 8 weeks than those who did not consume caffeine. While analyses revealed significance, the clinical significance should be investigated further. Research examining the effects of caffeine during strength training however, tends to focus on strength training performance as opposed to actual strength gains. For instance, acute caffeine consumption has been found to increase the number of repetitions completed before volitional failure, suggesting it may result in greater strength increases, however, not directly measured (Green, Wickwire, McLester, Gendle, Hudsen, Pritchett, Laurent, 2007; Woolf, Bidwell, \& Carlson, 2008). As the present study's finding is contrary to previously reported benefits of caffeine as an ergogenic aid, it prompted the use of caffeine to be disregarded.

Those who consumed caffeine also reported significantly higher RPE values for the kettlebell press than those who did not consume caffeine. Conversely, previous research 
supports that acute caffeine consumption increases performance while having no significant difference on reported RPEs compared to placebo trials (Green et al., 2007; Woolf et al., 2008). These studies included physiological measures of blood pressure (Woolf et al., 2008) and heart rate (Green et al., 2007; Woolf et al., 2008) which are commonly believed to increase with caffeine use and thereby increase perceived effort. Mean heart rate of both caffeine and placebo trials were not significantly different. Diastolic blood pressure was significantly raised during lifting bouts and systolic blood pressure was significantly raised after bouts during caffeine trials. Therefore, caffeine increased performance and blood pressure without producing significantly different RPE values than placebo trials (Green et al., 2007; Woolf et al., 2008). While the current study examined strength gains rather than performance, present RPE results are in opposition of previous examination of caffeine effects which prompted the disregard of present caffeine findings. It is important to note that since RPE is a subjective measure and there were no direct measures of exertion or fatigue in the present study, interpretation of these findings would only be conjecture.

\section{Related Literature}

The Variability of Practice literature that was generated by Schmidt's Schema theory is robust in support for the concept that practicing several variations of a task concurrently facilitates learning those tasks (Lai et al., 2000; McCracken \& Stelmach, 1977; Shea \& Kohl, 1990; 1991; Yao et al., 2009). The application of this concept to the acquisition of strength was not the original intent when these constructs were created. The application of cognitive processing theories were intended for new pattern acquisition and control, as well as the production of appropriate motor responses for 
skills involving cognitive control. However, as the strength and conditioning theorists have started using these constructs to predict strength acquisition, the question becomes whether or not the findings within Variability of Practice studies are generalizable to strength training settings.

Findings of the present study offer some support that Variability of Practice may facilitate strength gains. For instance, absolute strength gains in the leg press made by the variable treatment were significantly greater than the control group. While these gains were not significantly greater than those of the traditional treatment, the variable treatment's mean absolute and percent strength gains were greater over almost every time interval compared to the traditional and control groups. These results suggest dispersing volume and waving intensity within each training session, as done in the variable treatment, has some beneficial influence on strength acquisition of recreationally trained individuals. Findings also propose strength training programs with sets of lower intensity between those of higher intensity are perceived as less strenuous than those with constant sets of the same intensity. These lower RPEs are also associated with greater strength gains. This implies that greater strength can be acquired with programs designed to reduce perceived exertion levels, enabling inclusion of higher intensity sets. Together, these results provide support for the beneficial effects of Variability of Practice on strength acquisition. The facilitation of learning due to enhanced recall schema however, is not supported here as strength adaptations do not involve cognitive processing, but neuromechanical mechanisms. So while Variability of Practice design seems positively influence strength gains, it is not due to the same mechanisms as seen in motor learning and control research. 
It is difficult to compare the present study to previous strength research, as none that we are aware of have investigated variability within periodization to the same degree. Further research on "session undulating periodization" is required to compare effects on strength gains with linear and undulating forms of periodization. In Miranda et al. (2011), similar results were reported, as no significant differences in strength gains were found between the daily undulating periodized (DUP) treatment and the linear periodized (LP) treatment. Despite no significant differences in Miranda et al. (2011), the DUP treatment achieved a greater magnitude of strength gains. Due to this, increased variety in volume and intensity was recommended over traditional linear periodization. On the other hand, findings of Rhea et al. (2002) reported DUP as resulting in significantly greater percent strength gains in both the bench and leg press as well as significantly superior leg press absolute strength gains compared to the LP treatment. While DUP is the closest example of variable practice in published strength literature, examinations of LP versus undulating periodization (UP), provide insight on the influence of lower degrees of variability on strength gains.

One such study comparing LP and UP programs was Monteiro et al. (2009). In this particular investigation the UP treatment made significantly greater strength increases in the bench press and leg press compared to both the LP treatment and the non-periodized treatment. In Siamo et al. (2012) two of the four total upper body lifts examined had significantly higher $1 \mathrm{RM}$ values after UP treatment compared to LP. While the majority of strength studies recommend programming that alters volume and intensity to a greater degree than linear periodization, findings are not definitive. Commonly predicted factors reported by strength researchers contributing to insignificant results are small sample size 
and varying levels of experience within subjects. Further research accounting for commonly attributed limitations is needed.

\section{Limitations}

Future studies should focus on creating a greater difference in the degree of variability between treatments. Here, the change in volume and intensity occurred every three weeks for the traditional treatment due to the short 8 week timeframe of the study. Changes to program components of standard strength programs however, are not usually made earlier than four weeks. Consequently, on a continuum of constant to variable, the traditional treatment may not be as representative of constant practice as needed to demonstrate the effects of Variability of Practice on strength training. Additionally, as the greater volume of higher intensity sets in the variable treatment may be an important factor in superior strength gains, future studies should match intensity as well as relative volume.

The inability to monitor and ultimately control behavior of subjects outside training sessions would be a limitation in this study. While subjects were asked to refrain from consuming recreational substances 24 hours prior to sessions, subjects were not supervised at these times. Lack of sleep, dehydration, and malnutrition are possible performance reducing factors that were not monitored or accounted for by study administrators. As for control subjects, they agreed to maintain their current exercise regimen, a wide range of exercise modes were reported, among them weight lifting. The atmosphere created in a testing environment could also be a motivator resulting in improved 1RM values. As subjects were tested repeatedly, they naturally became more 
comfortable with the movement and protocol giving them a slight advantage over the previous testing sessions. Despite these possible reasons for the control group's increased strength, treatment groups did achieve greater relative strength in both lifts than control group at the end of the 8 weeks.

The inclusion of both male and female data introduces a wider range of relative baseline strength values within treatments. Therefore, a wider range of improvement capacities within treatments is created. Further variability within treatments was caused by allowing training subjects to continue aerobic exercise outside the training sessions. The variety of aerobic activities subjects participated in could result in differences in development and maintenance of muscle groups throughout the body. All of these factors could potentially affect strength related to the kettlebell and leg press. On the other hand, specificity of training states that strength improvements are specific to the posture, movement coordination, and sequence of contractions employed, which could be different for exercises seemingly similar (Folland \& Williams, 2007, p. 155).

\section{Conclusions}

Based on this study's findings, both variable and traditional treatment programs are viable and effective methods of strength training for recreationally trained males and females. Findings also support the beneficial effects of Variability of Practice on strength acquisition for a number of reasons. The variable treatment resulted in significantly greater strength increases compared to the control group while the traditional treatment did not, and variable treatment mean strength gains were greater than the traditional and control groups. RPE results revealed the most interesting treatment effects as 
significantly lower RPEs were found to be associated with greater strength gains. These findings suggests a strength training program designed to reduce exhaustion and fatigue would be more effective than one perceived as being more difficult. As the variable treatment resulted in lower RPEs and greater mean strength increases, a more variable program design would theoretically produce more advantageous results compared to a traditional program. Consequently, the greater mean strength gains and significantly lower RPEs of the variable strength program compel us to conclude it is a superior training method when compared to traditional periodization for strength acquisition.

\section{Recommendations}

To provide the most effective strength training program, increased variation as proposed by Pavel Tsatsouline and Dan John (2011) should be considered. By employing some form of variable training, the decrease in perceived exertion may also grant a degree of reduction in physical and mental strain, potentially decreasing incidence of training injuries. This is beneficial to athletes that require strength training in addition to conditioning and sport-specific practice. These athletes are doing the same movements repeatedly under greater and greater strain throughout the season. If exertion can somehow be reduced in the weight room, the risk of overtraining may decrease. This is important as overtraining can lead to decreased performance, musculoskeletal injury, or illness requiring athletes to cease training so they can recover. Ultimately this can throw them off track for an upcoming competition. Physical therapy settings may also apply, as patients who are experiencing pain or fatigue during rehab exercises would benefit from this type of program design. Similarly, older adults who experience difficulty or pain

during exercise who incorporate variable training concepts may achieve more positive 
improvements with less discomfort and effort than traditional training methods. Recent research examining optimal strength training for older adults supports the idea of using a program such as the variable treatment. Sets with lower repetitions and higher intensities are actually more effective for eliciting strength related adaptations in older populations (Granacher, Muehlbauer, Zahner, Gollhofer, \& Kressig, 2011).

The general population could benefit from variable training as well. While both males and females participate in resistance training, individuals who are already strong predominately make up the strength training population. Considering all the different physical activity options available, most people tend to choose other forms of exercise; and if they do resistance train it is not usually extensive enough to elicit significant strength increases. Strength decreases as a result of aging is a factor contributing to the loss of functional independence. Dependency related to strength losses occurs more frequently in individuals with lower strength levels while still living independently (Hernandez, Goldberg, \& Alexander, 2010; Shephard, 2008). By increasing strength training participation of the general population, the time individuals can continue to live independently increases (Harris, DeBeliso, Spitzer-Gibson, \& Adams, 2004; Shepard, 2008). The lower exertion levels experienced during the variable treatment is a potentially attractive attribute of variable training methods for these individuals. Research is inconclusive on what the optimal manipulation of program components is for older adults, but one thing is clear, strength training is effective for increasing strength and therefore functional abilities required to maintain independent living (Harris et al., 2004). Promoting variable strength training may increase participation and adherence to strength programs by individuals who are at risk for strength deficiency related dependency. 


\section{References}

American College of Sports Medicine. (2010). Thompson, W. R., Gordon, N. F., \& Pescatello, L. S. (Eds.). ACSM's Guidelines for Exercise Testing and Prescription (8th Ed.) (pp. 165 - 171). Baltimore, MD: Lippincott Williams \& Wilkins.

Aarskog, R., Wisnes, A., Wilhelmsen, K., Skogen, A., \& Bjordal, J. M. (2011). Comparison of two resistance training protocols, 6RM versus 12RM, to increase the 1RM in healthy young adults. A single-blind randomized controlled trial. Physiotherapy Research International, 17(3), 179-186.

Bird, S. P., Tarpenning, K., M., \& Marino, F. E. (2005). Designing resistance training programmes to enhance muscular fitness: a review of the acute programme variables. Sports Medicine, 35(10), 841-851.

Cormie, P., McGuigan, M. R., \& Newton, R. U. (2011). Developing maximal neuromuscular power: part 2 - training considerations for improving maximal power production. Sports Medicine, 41(2), 125-146.

Crewther, B., Keogh, J., Cronin, J., \& Cook, C. (2006). Possible stimuli for strength and power adaptation: acute hormonal responses. Sports Medicine, 36(3), 215-238.

Day, M. L., McGuigan, M. R., Brice, G., \& Foster, C. (2004). Monitoring exercise intensity during resistance training using the session RPE scale. Journal of Strength and Conditioning Research, 18(2), 353-358.

Duchateau, J., Semmler, J. G., \& Enoka, R. M. (2006). Training adaptations in the behavior of human motor units. Journal of Applied Physiology, 101, 1766-1775.

Enoka, R. M. (2002). Neuromechanics of Human Movement (3rd ed.). Champaign, IL: Human Kinetics.

Enoka, R. M. (2006) Neuromechanics of Human Movement (4th ed.). Champaign, IL: Human Kinetics.

Folland, J. P., \& Williams, A. G. (2007). The adaptations to strength training: morphological and neurological contributions to increased strength. Sports Med, $37(2), 145-168$.

Gabriel, D. A., Kamen, G., \& Frost, G. (2006) Neural adaptations to resistive exercise: mechanisms and recommendations for training practices. Sports Medicine, 36(2), 133-149.

Granacher, U., Muehlbauer, T., Zahner, L., Gollhofer, A., Kressig, R. W. (2011). Comparison of traditional and recent approaches in the promotion of balance and strength in older adults. Sports Medicine, 41(5), 377-400. 
Green, J. M., Wickwire, P. J., McLester, J. R., Gendle, S., Hudsen, G., Pritchett, R. C., \& Laurent, C. M. (2007). Effects of caffeine on repetitions to failure and ratings of perceived exertion during resistance training. International Journal of Sports Physiology and Performance, 2(3), 250-259.

Green, S., \& Sherwood, D. E. (2000). The benefits of random variable practice for accuracy and temporal error detection in a rapid aiming task. Research Quarterly for Exercise and Sport, 71(4), 398-402.

Goodwin, J. E., Eckerson, J. A., Grimes, C. R., \& Gordon, P. M. (1998). Effect of difference quantities of variable practice on acquisition, retention, and transfer of an applied motor skill. Perceptual and Motor SKills, 87(1), 147-151.

Hall, K. G. (1990). Variability of Practice and the Contextual Interference Effect in Motor Skill Acquisition. (Doctoral Dissertation). Louisiana State University, Baton Rouge, LA.

Harris, C., DeBeliso, M. A., Spitzer-Gibson, T. A., \& Adams, K. J. (2004). The effect of resistance-training on strength-gain response in the older adult. Journal of Strength and Conditioning Research, 18(4), 833-838.

Harrison, A. J., Keane, N. (2007). Effects of Variable and Fixed Practice on the Development of Jumping Ability in Young Children. Unpublished paper presented at XXV ISBS Symposium, Ouro Preto, Brazil.

Haudum, A., Birklbauer, J., Josef, K., \& Muller, E. (2011). Motor learning of Grossmotor skills under variable practice conditions. Ugdymas $\bullet$ Kuna Kultra $\bullet$ Sportas, 1(80), 22-28.

Heitman, R., Erdmann, J., Gurchiek, L., Kovaleski, J., \& Gilley, W. (1997). Constant versus variable practice in learning a motor task using individuals with learning disabilities. Clinical Kinesiology, 51(3), 62-65.

Hernandez, M. E., Goldberg, A., \& Alexander, N. B. (2010). Decreased muscle strength related to self-reported stooping, crouching, or kneeling difficulty in older adults. Physical Therapy, 90(1), 67-74.

Heyward, V. H. (2010). Advanced Fitness Assessment and Exercise Prescription (6th ed.). Champaign, IL: Human Kinetics.

Herodek, K., Simonovic, C., \& Rakovic, A. (2012). Periodization and strength training cycles. Activities in Physical Education and Sport, 2(2), 254-257.

John, D., \& Tsatsouline, P. (2011). Easy Strength. St. Paul, MN: Dragon Door Publications. 
Kantak, S. S., Sullivan, K. J., Fisher, B. E., Knowlton, B. J., \& Winstein, C. J. (2011). Transfer of motor learning engages specific neural substrates motor memory consolidation dependent on practice structure. Journal of Motor Behavior, 43(6), 499-506.

Kell, R. T. (2011). The influence of periodized resistance training on strength changes in men and women. The Journal of Strength and Conditioning Research, 25(3), 735744.

Lai, Q. Q., Shea, C. H., Wulf, G. G., \& Wright, D. L. (2000). Optimizing generalized motor program and parameter learning. Research Quarterly For Exercise \& Sport, 71(1), 10-24.

Lidor, R. R. (1995). The implementation of constant and variable - practices in skill acquisition: a field study. International Journal Of Physical Education, 32(4), 1116.

Matsouka, O., Trigonis, J., Simakis, S., Chavenetidis, K., \& Kioumourjoglou, E. (2010). Variability of practice and enhancement of acquisition, retention and transfer of learning using an outdoor throwing motor skill by children with intellectual disabilities. Studies in Physical Culture \& Tourism, 17(2), 157-164.

McCracken, H. D. \& Stelmach G. E. (1977). A test of schema theory of discrete motor learning. Journal of Motor Behavior, 9(3), 193-200.

Memmert, D. (2006). Long-term effects of type of practice on the learning and transfer of a complex motor skill. Perceptual and Motor Skills, 103(3), 912-916.

Miranda, F., Simao, R., Rhea, M., Bunker, D., Prestes, J., Leite, R., \& ... Novaes, J. (2011). Effects of linear vs. daily undulatory periodized resistance training on maximal and submaximal strength gains. Journal Of Strength \& Conditioning Research, 25(7), 1824-1830.

Monteiro, A. G., Aoki, M. S., Evangelista, A. L., Alveno, D. A., Monteiro, G. A., Picarro, I. D., \& Ugrinowitsch, C. (2009). Nonlinear periodization maximizes strength gains in split resistance training routines. . Journal Of Strength \& Conditioning Research, 23(4), 1321-1326.

Ranganathan, R., \& Newell, K. M. (2010). Motor Learning through Induced Variability at the Task Goal and Execution Redundancy Levels. Journal Of Motor Behavior, 42(5), 307-316.

Rhea, M. R., Ball, S. D., Phillips, W. T., Burkett, L. N. (2002). A comparison of linear and daily undulating periodized programs with equated volume and intensity for strength. Journal of Strength \& Conditioning Research, 16(2), 250-255. 
Rhea, C. K., Wutzke, C. J., \& Lewek, M. D. (2012). Gait dynamics following variable and constant speed gait training in individuals with chronic stroke. Gait \& Posture, 36(2), 332-334.

Schmidt, R. A. (1975). A schema theory of discrete motor skill learning. Psychological Review, 82, 225-260.

Schmidt, R. A. (1988). Motor control and learning; A behavioral emphasis (2nd ed.). Champaign, IL: Human Kinetics

Schmidt, R. A., \& Lee, T. D. (2005). Motor Control and Learning (4th ed.). Champaign, IL: Human Kinetics.

Shea, C. H., Kohl, R. M. (1990). Specificity and Variability of Practice. Research Quarterly for Exercise and Sport, 61(2), 169-177.

Shea, C. H., Kohl, R. M. (1991). Composition of Practice - Influence on the Retention of Motor Skills. Research Quarterly for Exercise and Sport, 62(2), 187-195.

Shea, C. H., Lai, Q. Q., Wright, D. L., Immink, M. M., \& Black, C. C. (2001). Consistent and variable practice conditions: effects on relative and absolute timing. Journal of Motor Behavior, 33(2), 139-152.

Shephard, R. J. (2008). The importance of oxygen transport, strength and flexibility in maintaining independence of the elderly. Medicina Sportiva, 12(4), 165-174.

Simäo, R., Spineti, J., Freitas de Salles, B., Matta, T., Fernandes, L., Fleck, S. J., \& ... Strom-Olsen, H. E. (2012). Comparison between nonlinear and linear periodized resistance training: hypertrophic and strength effects. Journal Of Strength \& Conditioning Research 26(5), 1389-1395.

Tsatsouline, P. (2006). Enter the Kettlebell! St. Paul, MN: Dragon Door Publications, Inc.

Van Rossum, J. H. A. (1990). Schmidt's schema theory: the empirical base the variability of practice hypothesis. Human Movement Science, 9, 387-435.

Whitacre, C. A., \& Shea, C. H. (2000). Performance and learning of generalized motor programs: relative (GMP) and absolute (parameter) errors. Journal of Motor Behavior, 32(2), 163-175.

Woolf, K., Bidwell, W. K., \& Carlson, A. G. (2008). The effect of caffeine as an ergogenic aid in anaerobic exercise. International journal of Sport Nutrition and Exercise Metabolism, 18(4), 412-429. 
Yao, W. X., DeSola, W., \& Bi, Z. C. (2009). Variable practice versus constant practice in the acquisition of wheelchair propulsive speeds. Perceptual and Motor Skills, 109(1), 133-139. 